\title{
Lifting, Superadditivity, Mixed Integer Rounding and Single Node Flow Sets Revisited
}

\author{
Quentin Louveaux, Laurence A. Wolsey ${ }^{1 \star}$ \\ CORE and INMA, Université Catholique de Louvain, Louvain-la-Neuve \\ Received: date / Revised version: date
}

Corresponding author: L.A. Wolsey, CORE, Voie du Roman Pays, 1348 Louvainla-Neuve, Belgium

Email: louveaux@core.ucl.ac.be,wolsey@core.ucl.ac.be

\begin{abstract}
In this survey we attempt to give a unified presentation of a variety of results on the lifting of valid inequalities, as well as a standard procedure combining mixed integer rounding with lifting for the development of strong valid inequalities for knapsack and single node flow sets. Our hope is that the latter can be used in practice to generate cutting planes for mixed integer programs.

The survey contains essentially two parts. In the first we present lifting in a very general way, emphasizing superadditive lifting which allows one to lift simultaneously different sets of variables. In the second, our procedure for generating strong valid inequalities consists of reduction to a knapsack set with a single continuous variable, construction of a mixed integer rounding inequality, and superadditive lifting. It is applied to several generalizations of the 0-1 single node flow set.
\end{abstract}

Keywords: Lifting, Mixed Integer Rounding, Single Node Flow Sets Classification: $90 \mathrm{C} 11,90 \mathrm{C} 27$

\footnotetext{
* Present address: Core, Voie du Roman Pays 34, 1348 Louvain-la-Neuve, Belgium. The first author is supported by the FNRS as a research fellow. This paper presents research results of the Belgian Program on Interuniversity Poles of Attraction initiated by the Belgian State, Prime Minister's Office, Science Policy Programming. The scientific responsibility is assumed by the authors.

This research was also supported by the European Commission GROWTH Programme, Research Project LISCOS, Large Scale Integrated Supply Chain Optimization Software Based on Branch-andCut and Constraint Programming Methods, Contract No. GRDI-1999-10056, and the project TMRDONET nr. ERB FMRX-CT98-0202.
} 


\section{Introduction}

Considerable work has been carried out since the early 1970s in generating strong valid inequalities using "lifting" as introduced by Padberg (1973). In particular lifting has been crucial in developing strong facet-defining inequalites for 0-1 knapsack sets Balas (1975); Hammer et al. (1975); Wolsey (1975), and their mixed integer counterpart, called single node flow sets Gu et al. (1999); Padberg et al. (1985); Stallaert (1997); Van Roy and Wolsey (1986). What is more these inequalities have provided effective cuts for 0-1 programs Crowder et al. (1963) and mixed 0-1 programs Van Roy and Wolsey (1987), and along with Gomory mixed integer cuts Gomory (1960) and mixed integer rounding inequalities Nemhauser and Wolsey (1990) form part of state-of-the-art commercial mixed integer programming systems such as Cplex and Xpress.

Our goal here is to revisit some of this work, in particular that concerning lifting and the generation of valid inequalities for single node flow sets.

Without being exhaustive, we aim to touch at least indirectly on the work on cover inequalities for 0-1 knapsack problems and on flow cover inequalities for single node flow sets referenced above, work on variable lifting (Padberg, 1973; Wolsey, 1976) and superadditive lifting (Gu et al., 2000; Wolsey, 1977), work on knapsack problems with integer and/or continuous variables (Atamtürk, 2002; Ceria et al., 1998; Marchand and Wolsey, 1999; Richard et al., 2002a), and on some models generalizing the knapsack and single node flow sets (Atamtürk et al., 2001; Goemans, 1989; Miller et al., 2003b; Wolsey, 1990).

The viewpoint taken is close to that in Gu et al. $(1999,2000)$ and Marchand and Wolsey $(1999,2001)$ dealing with single node flow sets, simultaneous lifting, knapsack sets with a continuous variable and mixed integer rounding respectively. First we develop fairly general results on the lifting of sets of variables, and then, turning specifically to single node flow sets, we show how mixed integer rounding combined with lifting provides a unified and computationally simple way to obtain many of the valid inequalities for mixed 0-1 sets that have been proposed in the literature, as well as new inequalities for integer single node flow sets.

We now describe the contents of this paper. In Section 2 we take an abstract view of the lifting problem and of the question how to generate valid inequalities for one set from the valid inequalities of a second lower-dimensional subset, or of a second higher-dimensional set.

In Section 3 we present the problem of lifting a valid inequality to a valid inequality for a higher dimensional set in some detail. Examples are presented to suggest some new computational possibilities. We also examine the crucial role of superadditivity in simplifying the calculations, and allowing simultaneous lifting of several groups of variables. 
We then turn to the single node flow set, denoted $X^{N}\left(n_{1}, n_{2}, b, a, u\right)$,

$$
\begin{gathered}
\sum_{j \in N_{1}} x_{j}-\sum_{j \in N_{2}} x_{j}-s \leq b \\
x_{j} \leq a_{j} y_{j} \text { for } j \in N_{1} \cup N_{2} \\
y_{j} \leq u_{j} \text { for } j \in N_{1} \cup N_{2} \\
x \in \mathbb{R}_{+}^{n_{1}+n_{2}}, y \in \mathbb{Z}_{+}^{n_{1}+n_{2}}, s \in \mathbb{R}_{+}^{1}
\end{gathered}
$$

where $n_{1}=\left|N^{1}\right|, n_{2}=\left|N^{2}\right|, n=n_{1}+n_{2}, b \in \mathbb{Z}^{1}, a \in \mathbb{R}^{n}, u \in \mathbb{Z}_{+}^{n}$. In Section 4 , we consider the 0-1 case. Such sets were initially studied as a natural mixed integer generalization of 0-1 knapsack sets, see Remark 2 below, and because essentially every mixed integer row can be rewritten as a single node flow set, see p286 in Nemhauser and Wolsey (1988). Here we show that the standard flow cover inequality derived in Van Roy and Wolsey (1986) can be strengthened by using mixed integer rounding, and/or superadditive lifting. In addition we indicate how the reverse cover/flow pack inequalities of Stallaert (1997) and Atamtürk (2001) can be derived as flow cover inequalities by reversing arc directions in the single node flow set.

In Section 5, we consider the general case when the integer variables are bounded, but not 0-1. An integer version of the mixed integer rounding flow cover inequality is derived, and results of Atamtürk (2002) for the corresponding knapsack set are used to strengthen the inequalities.

In Section 6 we consider three generalizations of $X^{N}$, and examine to what extent they can be treated using our standard approach (Atamtürk et al., 2001; Miller et al., 2003a; Wolsey, 1990). We terminate by mentioning a few open questions.

\section{Generating Valid Inequalities from Sub or Supersets}

Throughout we consider mixed integer sets described by integer (or rational) coefficients of the form

$$
X=\left\{z \in \mathbb{R}_{+}^{n^{1}} \times \mathbb{Z}_{+}^{n^{2}}: A z \leq b\right\}
$$

with $n=n^{1}+n^{2}$. Here we also consider a second lower-dimensional subset

$$
Y=X \cap\{z: C z=e\}
$$

Below we consider two possibilities: using knowledge about $\operatorname{conv}(X)$ to get complete information about $\operatorname{conv}(Y)$, or using $\operatorname{conv}(Y)$ to get partial information about $\operatorname{conv}(X)$. 


\subsection{Valid Inequalities from Supersets}

Remark 1. If $C z \leq e$ for all $z \in X$, then $\operatorname{conv}(Y)$ is a face of $\operatorname{conv}(X)$, and

$$
\operatorname{conv}(Y)=\operatorname{conv}(X) \cap\{z: C z=e\} .
$$

Thus every facet-defining inequality of $\operatorname{conv}(Y)$ corresponds to a facet-defining inequality of $\operatorname{conv}(X)$.

In both examples presented below, the superset $X$ is the $0-1$ single node flow set $X^{N}$ with $u=\underline{1}$.

Remark 2. The 0-1 knapsack set with a single continuous variable (Marchand and Wolsey, 1999)

$$
X^{C K}=\left\{(y, s) \in\{0,1\}^{n} \times \mathbb{R}_{+}^{1}: \sum_{j \in N_{1}} a_{j} y_{j} \leq b+s\right\}
$$

is obtained from the single node flow set $X^{N}$ with $n_{1}=n, n_{2}=0$ by setting $x_{j}=a_{j} y_{j}$ for all $j \in N_{1}$.

Remark 3. The 0-1 single node flow model with some simple bounds Richard et al. (2002a,b)

$$
\begin{gathered}
X^{N C}=\left\{(x, y, s) \in \mathbb{R}_{+}^{n} \times \mathbb{Z}^{n-r} \times \mathbb{R}_{+}^{1}:\right. \\
\sum_{j \in N_{1}} x_{j}-\sum_{j \in N_{2}} x_{j} \leq b+s \\
x_{j} \leq a_{j} y_{j}, y_{j} \leq 1 \text { for } j \in\left(N_{1} \backslash R_{1}\right) \cup\left(N_{2} \backslash R_{2}\right) \\
\left.x_{j} \leq a_{j} \text { for } j \in R_{1} \cup R_{2}\right\},
\end{gathered}
$$

is obtained from $X^{N}$ by setting $y_{j}=1$ for $j \in R_{1} \cup R_{2}$, where $r=\left|R_{1} \cup R_{2}\right|$.

Example 1. Consider the set $X^{N}(3,2,4,(3,4,5,2,3), \underline{1})$, or

$$
\begin{gathered}
x_{1}+x_{2}+x_{3}-x_{4}-x_{5} \leq 4+s \\
x_{1} \leq 3 y_{1}, x_{2} \leq 4 y_{2}, x_{3} \leq 5 y_{3}, x_{4} \leq 2 y_{4}, x_{5} \leq 3 y_{5} \\
x \in \mathbb{R}_{+}^{5}, y \in\{0,1\}^{5}, s \in \mathbb{R}_{+}^{1}
\end{gathered}
$$

shown in Figure 1.

Now consider the 0-1 knapsack set with a single continuous variable $X^{C K}$

$$
\begin{gathered}
3 y_{1}+4 y_{2}+5 y_{3}-2 y_{4}-3 y_{5} \leq 4+s \\
y \in\{0,1\}^{5}, s \in \mathbb{R}_{+}^{1} .
\end{gathered}
$$

A facet of $\operatorname{conv}\left(X^{C K}\right)$, such as that represented by the inequality

$$
2 y_{1}+3 y_{2}+4 y_{3}-y_{4}-3 y_{5} \leq 3+s,
$$




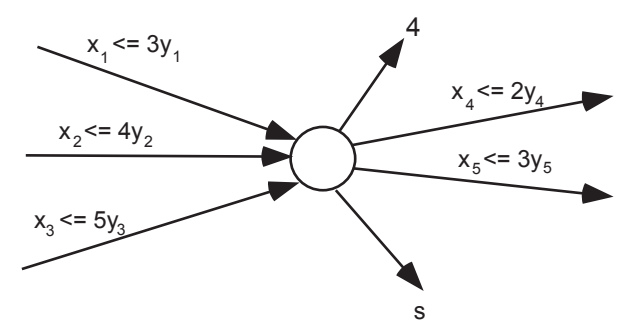

Fig. 1. 0-1 Single Node Flow Set

can be obtained directly from the facet

$$
x_{1}-y_{1}+x_{2}-y_{2}+x_{3}-y_{3}-y_{4}-x_{5} \leq 3+s
$$

of $\operatorname{conv}\left(X^{N}\right)$ by setting $x_{1}=3 y_{1}, x_{2}=4 y_{2}, x_{3}=5 y_{3}$ and $x_{5}=3 y_{5}$.

Another consequence of Remark 1 concerns the separation problem arising when one wishes to find a valid inequality for $X$ cutting off a point $z^{*}$.

Remark 4. If $C z \leq e$ for all $z \in X$ and $z^{*}$ satisfies $C z^{*}=e$, then $z^{*} \notin \operatorname{conv}(X)$ if and only if $z^{*} \notin \operatorname{conv}(Y)$.

Thus to solve the separation problem over $X$, it suffices to solve the separation problem over $Y$, and then convert the violated valid inequality for $Y$ into a violated inequality for $X$. The latter conversion is precisely the lifting problem that we now consider.

\subsection{Valid Inequalities from Subsets}

Here we ask when a valid inequality for $Y$ can be "lifted" into a valid inequality for $X$. We distinguish two cases: either $C z \leq e$ for all $z \in X$, i.e. $\operatorname{conv}(Y)$ is a face of $\operatorname{conv}(X)$, as above, or not.

Remark 5. If $\operatorname{conv}(Y)$ is a face of $\operatorname{conv}(X)$ and $\pi^{1} z \leq \pi_{0}$ is a valid inequality for $\operatorname{conv}(Y)$, there exists a vector $\pi^{2}$ such that

$$
\pi^{1} z+\pi^{2}(e-C z) \leq \pi_{0}
$$

is valid for $\operatorname{conv}(X)$.

It is easily verified that the inequality is valid if one takes $\pi_{j}^{2}=-M$ for all $j \in N$ with $M$ sufficiently large.

On the other hand, when $C z=e$ cuts through the interior of $\operatorname{conv}(X)$, it is known that the required multipliers may not exist. This is demonstrated by the following simple example. 
Example 2. Take the integer knapsack set

$$
X=\left\{y \in \mathbb{Z}_{+}^{2}: 3 y_{1}+5 y_{2} \leq 21, y_{2} \leq 4\right\},
$$

and let

$$
Y=X \cap\left\{y \in \mathbb{R}^{2}: y_{2}=2\right\}=\left\{\left(y_{1}, 2\right) \in \mathbb{Z}_{+}^{2}: 3 y_{1} \leq 11\right\}
$$

with valid inequality $y_{1} \leq 3$. Now if $y_{1}+\pi^{2}\left(2-y_{2}\right) \leq 3$ is a valid inequality for $X$, the point $(0,4) \in X$ implies $\pi^{2} \geq-3 / 2$, whereas the point $(7,0) \in X$ implies that $\pi^{2} \leq-2$. Thus there is no possible multiplier.

In the next section we consider how to find the multipliers $\pi^{2}$ or show that there are none. However the question is posed a little differently. Specifically, consider introducing slack variables $t=e-C z \geq 0$. Now $Y$ is obtained from $X$ by setting $t=0$, and the problem is to find lifting coefficients $\pi^{2}$ so that $\pi^{1} z+\pi^{2} t \leq \pi_{0}$ is valid.

\section{Lifting Valid Inequalities}

We consider the mixed integer sets, denoted $Z^{\tau}(b)$, of the form

$$
\begin{gathered}
\sum_{k=1}^{\tau} A^{k} z^{k} \leq b+s \\
z^{k} \in X^{k} \text { for } k=1, \ldots \tau, s \in \mathbb{R}_{+}^{m},
\end{gathered}
$$

where $A^{k} \in \mathbb{R}^{m \times n_{k}}$ for $k=1, \ldots, K, b \in \mathbb{R}^{m}, X^{k}=\left\{z^{k} \in \mathbb{R}^{n_{k}^{1}} \times \mathbb{Z}^{n_{k}^{2}}\right.$ : $\left.C^{k} z^{k} \leq c^{k}\right\}$ with $n_{k}=n_{k}^{1}+n_{k}^{2}$ is a mixed integer set in $\mathbb{R}^{n_{k}}$ for all $k$ and $0 \in X^{k}$ for $k=2, \ldots, K$. Here we study how to find valid inequalities for $Z^{K}(b)$, starting from valid inequalities for $Z^{1}(b)$.

The simultaneous lifting of variables in blocks was presented by $\mathrm{Gu}$ et al. (2000). The specific calculations required to lift flow cover inequalities with each block consisting of two variables $\left(x_{k}, y_{k}\right)$ were presented in Gu et al. (1999). The description below is new in several respects: we reduce to a single case by imposing that the variables are fixed to zero $\left(z^{k}=0\right)$ which may imply a change of variables, and we show that the structure of the lifting functions (piecewise linear) and the sets of valid coefficients (polyhedral) is such that the calculations are well-defined.

The lifting approach consists of the following:

1. Fix $z^{k}=0$ for $k=2, \ldots, K$.

2. Find a tight valid inequality $\pi^{1} z^{1} \leq \pi_{0}+\nu s$ for $Z^{1}(b)$. 
3. Iterations $\tau=2, \ldots, K$. Given a tight valid inequality $\sum_{k=1}^{\tau-1} \pi^{k} z^{k} \leq \pi_{0}+\nu s$ for $Z^{\tau-1}(b)$, lift the variables $z^{\tau}$ and derive coefficients $\pi^{\tau}$ such that

$$
\sum_{k=1}^{\tau-1} \pi^{k} z^{k}+\pi^{\tau} z^{\tau} \leq \pi_{0}+\nu s
$$

is valid for $Z^{\tau}(b)$, or determine that no such $\pi^{\tau}$ exists.

Relative to the description in Section 2, $X=Z^{K}(b)$ and $Y=Z^{K}(b) \cap\left\{\left(z^{1}, \ldots, z^{K}\right)\right.$ : $\left.z^{2}=\ldots=z^{K}=0\right\}=Z^{1}(b)$.

Below we discuss theoretically how to find valid lifting coefficients $\pi^{\tau}$ for $\tau=$ $2, \ldots, K$, and then consider cases in which the required calculations may be tractable.

\subsection{Lifting: Basic Theory}

We first define a crucial function.

Definition 1. The lifting function $\phi^{k}: \mathbb{R}^{m} \rightarrow \mathbb{R}^{1}$ is

$$
\phi^{k}(u)=\min \left\{\pi_{0}+\nu s-\sum_{\tau=1}^{k} \pi^{\tau} z^{\tau}:\left(z^{1}, \ldots, z^{k}, s\right) \in Z^{k}(b-u)\right\}
$$

Note that for any $u \in \mathbb{R}^{m}$ and any $\left(z^{1}, \ldots, z^{k}\right) \in X^{1} \times \ldots \times X^{k}$, there exists $s \in \mathbb{R}_{+}^{m}$ such that $\left(z^{1}, \ldots, z^{k}, s\right) \in Z^{k}(b-u)$, so $\phi^{k}(u)$ is finite for all $u \in \mathbb{R}^{m}$. Also from the definition, it follows that

$$
\phi^{1} \geq \ldots \geq \phi^{K}
$$

We also introduce the set

$$
\Pi^{k}=\left\{\pi \in \mathbb{R}^{n_{k}}: \pi t \leq \phi^{k-1}\left(A^{k} t\right) \text { for all } t \in X^{k}\right\}
$$

of lifting coefficients. For most of the results, it will suffice to consider the case where $K=2$.

Proposition 1. If $\pi^{1} z^{1} \leq \pi_{0}+\nu$ s is a tight valid inequality for $Z^{1}(b)$,

$$
\pi^{1} z^{1}+\pi^{2} z^{2} \leq \pi_{0}+\nu s
$$

is valid for $Z^{2}(b)$ if and only if $\pi^{2} \in \Pi^{2}$. 
Proof. Suppose $\pi^{2} \in \Pi^{2}$ and consider a point $\left(\bar{z}^{1}, t, \bar{s}\right) \in Z^{2}(b)$. Then

$$
\begin{gathered}
\pi^{2} t \leq \phi^{1}\left(A^{2} t\right) \text { as } t \in X^{2} \\
\leq \pi^{0}+\nu \bar{s}-\pi^{1} \bar{z}^{1} \text { as }\left(\bar{z}^{1}, \bar{s}\right) \in Z^{1}\left(b-A^{2} t\right)
\end{gathered}
$$

and so the inequality is valid.

Conversely if $\pi^{2} t>\phi^{1}\left(A^{2} t\right)$ for some $t \in X^{2}$, take a point $\left(z^{1}, s\right) \in Z^{1}(b-$ $\left.A^{2} t\right)$ with $\pi^{1} z^{1}=\pi_{0}+\nu s-\phi^{1}\left(A^{2} t\right)$. Now $\left(z^{1}, t, s\right) \in Z^{2}(b)$, but $\pi^{1} z^{1}+\pi^{2} t>$ $\pi_{0}+\nu s$, and the inequality is not valid.

We now consider briefly the structure of the functions $\phi^{k}$ and the sets $\Pi^{k}$, and whether the required calculations can be carried out.

Remark 6. $\phi^{k}(u)$ is the value function of a mixed integer program. Thus there exists a finite set of polyhedra $P^{q}=\left\{u \in \mathbb{R}^{m}: D^{q} u \leq d^{q}\right\}$ whose union is $\mathbb{R}^{m}$ and vectors $\left(\alpha^{q}, \beta^{q}\right) \in \mathbb{R}^{m} \times \mathbb{R}^{1}$ such that for all $q$

$$
\phi^{k}(u)=\alpha^{q} u+\beta^{q} \text { for } u \in P^{q} .
$$

Proposition 2. If each set $X^{k}=\left\{(x, y) \in \mathbb{R}_{+}^{n_{k}^{1}} \times \mathbb{Z}_{+}^{n_{k}^{2}}: C_{1}^{k} x+C_{2}^{k} y \leq c^{k}\right\}$ is a bounded mixed integer set, then $\Pi^{k}$ is a polyhedron.

Proof We consider $\Pi^{2}$. Now for fixed $y \in \operatorname{proj}_{y}\left(X^{2}\right)$ and fixed region $q$, with $\pi=(\lambda, \mu) \in \Pi^{2}$ and $t=(x, y) \in X^{2}, \pi \in \Pi^{2}$ if and only if

$$
\lambda x+\mu y \leq \phi^{2}\left(A^{2} t\right) \leq \alpha^{q}\left(A_{1}^{2} x+A_{2}^{2} y\right)+\beta^{q}
$$

for all $u=A^{2} t=A_{1}^{2} x+A_{2}^{2} y$ satisfying $D^{q} u \leq d^{q}$, with $A^{2}=\left(A_{1}^{2}, A_{2}^{2}\right)$ and $C^{2}=\left(C_{1}^{2}, C_{2}^{2}\right)$.

In other words $\pi \in \Pi^{2}$ if and only if $\left(\lambda-\alpha^{q} A_{1}^{2}\right) x+\left(\mu-\alpha^{q} A_{2}^{2}\right) y \leq \beta^{q}$ for all $x$ such that $D^{q}\left(A_{1}^{2} x+A_{2}^{2} y\right) \leq d^{q}, C_{1}^{2} x \leq c-C_{2}^{2} y, x \geq 0$. For fixed $y$ and $q$, the latter set is a bounded polyhedron in $x$ with a finite number of extreme points $\left\{x^{t}\right\}_{t=1}^{T}$. Thus enumerating over the finite set of feasible integer vectors $y$, the finite number of regions $q$ and the finite set of extreme points, we obtain an explicit description:

$$
\Pi^{2}=\left\{\pi=(\lambda, \mu): \lambda x^{t}+\mu y \leq \alpha^{q}\left(A_{1}^{2} x^{t}+A_{2}^{2} y\right)+\beta^{q} \forall q, y, t\right\} .
$$

Next if $\Pi^{2} \neq \emptyset$ and lifting coefficients $\pi^{2} \in \Pi^{2}$ have been selected, one needs to calculate the new lifting function $\phi^{2}$. Rather than calculating it from scratch, it can also be obtained by updating.

Proposition 3.

$$
\phi^{2}(u)=\min _{t \in X^{2}}\left[\phi^{1}\left(u+A^{2} t\right)-\pi^{2} t\right]
$$


Proof. By definition $\phi^{2}(u)$

$$
\begin{gathered}
=\min _{z^{1}, z^{2}, s}\left\{\pi_{0}+\nu s-\pi^{1} z^{1}-\pi^{2} z^{2}:\right. \\
\left.A^{1} z^{1}+A^{2} z^{2} \leq b+s-u, z^{i} \in X^{i} \text { for } i=1,2, s \geq 0\right\} \\
=\min _{t \in X^{2}}\left\{\operatorname { m i n } _ { z ^ { 1 } , s } \left\{\pi_{0}+\nu s-\pi^{1} z^{1}:\right.\right. \\
\left.\left.A^{1} z^{1} \leq b+s-u-A^{2} t, z^{1} \in X^{1}, s \geq 0\right\}-\pi^{2} t\right\} \\
=\min _{t \in X^{2}}\left\{\phi^{1}\left(u+A^{2} t\right)-\pi^{2} t\right\} .
\end{gathered}
$$

Example 3. (The Lifting Function and the Set of Lifting Coefficients).

Consider the set

$$
\begin{gathered}
5 y_{1}+5 y_{2}+5 y_{3}+x_{4}+2 y_{4} \leq 12+s \\
1 y_{4} \leq x_{4} \leq 3 y_{4} \\
y_{i} \in\{0,1\} \text { for } i=1, \ldots 3, y_{4} \in\{0,1,2\}, x_{4} \in \mathbb{R}_{+}^{1} .
\end{gathered}
$$

This can be modelled in the form (1) with $A^{1}=(5,5,5), A^{2}=(1,2), b=$ $12, X^{1}=\{0,1\}^{3}, X^{2}=\left\{\left(x_{4}, y_{4}\right) \in \mathbb{R}_{+}^{1} \times \mathbb{Z}_{+}^{1}: 1 y_{4} \leq x_{4} \leq 3 y_{4}, y_{4} \leq 2\right\}$.

As valid inequality for $Z^{1}(b)$, we take

$$
3 y_{1}+3 y_{2}+3 y_{3} \leq 6+s .
$$

The lifting function $\phi^{1}$ is given, see Figure 2, by

$$
\phi^{1}(u)=\left\{\begin{array}{ccc}
-3 & \text { if } & u \leq-3 \\
u & \text { if } & -3 \leq u<0 \\
0 & \text { if } & 0 \leq u<2 \\
u-2 & \text { if } & 2 \leq u<5 \\
3 & \text { if } & 5 \leq u<7 \\
u-4 & \text { if } & 7 \leq u<10 \\
6 & \text { if } & 10 \leq u<12 \\
u-6 & \text { if } & 12 \leq u
\end{array}\right.
$$

Now we wish to find lifting coefficients $\pi^{2}=(\lambda, \mu) \in \Pi^{2}$. We note that $3 \leq$ $x_{4}+2 y_{4} \leq 10$ and $y_{4} \in\{1,2\}$ for $(x, y) \in X^{2} \backslash\{(0,0)\}$.

For $y_{4}=1,1 \leq x_{4} \leq 3$ and thus $3 \leq u=x_{4}+2 y_{4} \leq 5$. This just intersects the region/segment $u \in[2,5]$, and the extreme points are $x_{4}=1$ and $x_{4}=3$.

For $y_{4}=2,6 \leq u=x_{4}+2 y_{4} \leq 10$, and two segments [5,7] and [7,10] are intersected leading to the extreme points $x_{4}=1, x_{4}=3$ and $x_{4}=6$. 


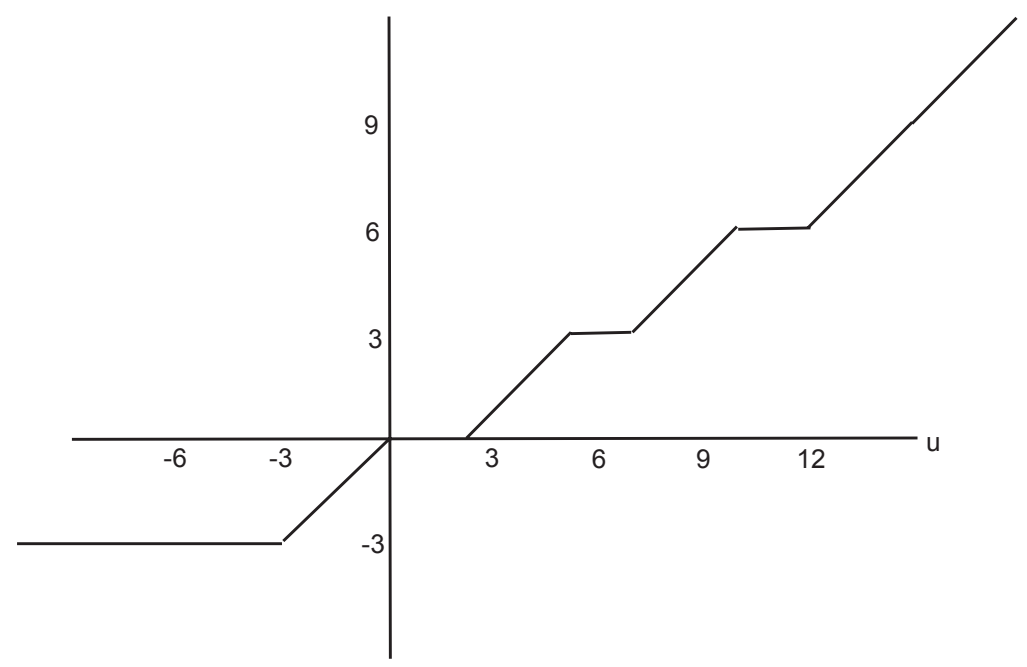

Fig. 2. Lifting function

So $\Pi^{2}$ is described by the inequalities

$$
\begin{aligned}
& 1 \lambda+1 \mu \leq \phi^{1}(3)=1 \\
& 3 \lambda+1 \mu \leq \phi^{1}(5)=3 \\
& 1 \lambda+2 \mu \leq \phi^{1}(5)=3 \\
& 3 \lambda+2 \mu \leq \phi^{1}(7)=3 \\
& 6 \lambda+2 \mu \leq \phi^{1}(10)=6
\end{aligned}
$$

with extreme points $\pi=(-1,2)$ and $\pi=(1,0)$ giving the valid inequalities

$$
\begin{gathered}
3 y_{1}+3 y_{2}+3 y_{3}-z_{4}+2 y_{4} \leq 12+s \text { and } \\
3 y_{1}+3 y_{2}+3 y_{3}+z_{4} \leq 12+s . \square
\end{gathered}
$$

\subsection{Superadditive Lifting}

Lifting requires calculation of the lifting function $\phi^{k-1}(u)$ and then finding a point $\pi^{k} \in \Pi^{k}$ for $k=2, \ldots, K$. Calculation of $\phi^{k-1}(u)$ is typically a difficult problem, so in practice there is a need to reduce the amount of computation. When the lifting function has appropriate structure, more can be said and the amount of computation can be reduced.

Definition 2. A function $F: D \rightarrow \mathbb{R}$ is superadditive on $D \subseteq \mathbb{R}^{m}$ if

$$
F(u)+F(v) \leq F(u+v)
$$

for all $u, v$ for which $u, v, u+v \in D$. 
Throughout we will assume that $D$ is a cone, so that $u, v \in D$ implies $u+v \in D$. We also limit our attention to superadditive functions that are continuous, with the property that $\bar{F}(d)=\lim _{t \rightarrow 0} \frac{F(t d)}{t}$ exists for all $d \in D$, and with $F(\underline{0})=0$.

Two classes of functions will be very useful later.

Definition 3. For $0<\alpha<1$, the mixed integer rounding function $F_{\alpha}: \mathbb{R}^{1} \rightarrow \mathbb{R}^{1}$ is defined by

$$
F_{\alpha}(d)=\lfloor d\rfloor+\frac{\left(f_{d}-\alpha\right)^{+}}{1-\alpha}
$$

where $f_{d}=d-\lfloor d\rfloor$.

This function is superadditive on $\mathbb{R}^{1}$ and is shown in Figure 3 . Note that $\bar{F}_{\alpha}$ exists, and $\bar{F}_{\alpha}(d)=\min \left[0, \frac{d}{1-\alpha}\right]$.

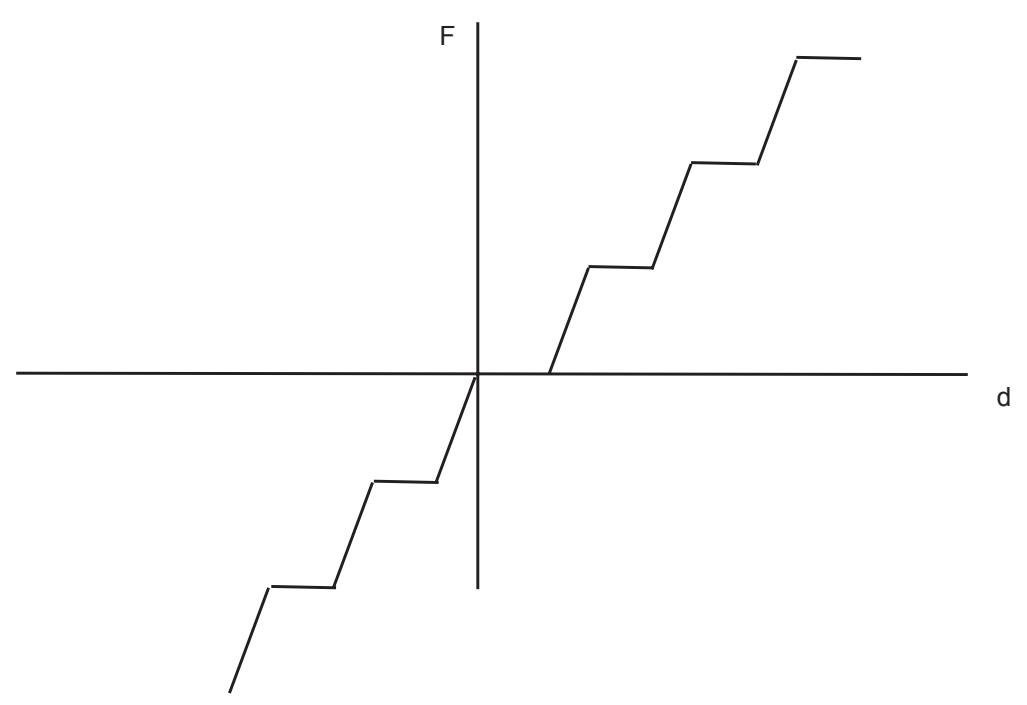

Fig. 3. Superadditive MIR Function

Definition 4. Suppose that $a \in \mathbb{R}_{+}^{n}$ with $a_{i_{1}} \geq a_{i_{2}} \geq \ldots \geq a_{i_{r}}>\lambda \geq$ $a_{i_{r+1}} \ldots a_{i_{n}}>0$, and let $A_{t}=\sum_{j=1}^{t} a_{i_{j}}$ for $t \leq r$ with $A_{0}=0$ and $A_{r+1}=\infty$. Define $G_{a, \lambda}: \mathbb{R}_{+}^{1} \rightarrow \mathbb{R}_{+}^{1}$ by

$$
G_{a, \lambda}(u)=\left\{\begin{array}{l}
(j-1) \lambda \text { if } A_{j-1} \leq u \leq A_{j}-\lambda j=1, \ldots, r \\
(j-1) \lambda+\left[u-\left(A_{j}-\lambda\right)\right] \text { if } A_{j}-\lambda \leq u \leq A_{j} j=1, \ldots, r-1 \\
(r-1) \lambda+\left[u-\left(A_{r}-\lambda\right)\right] \text { if } A_{r}-\lambda \leq u
\end{array}\right.
$$




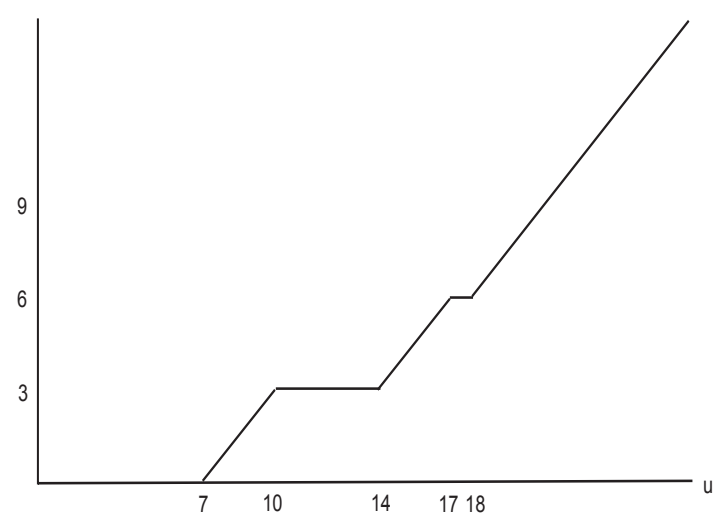

Fig. 4. Superadditive Function $G_{a, \lambda}$ on $\mathbb{R}_{+}^{1}$

This function is superadditive on $\mathbb{R}_{+}^{1}$ and an instance with $a=(10,7,4,2)$ and $\lambda=3$ is shown in Figure 4.

Though we will not use it directly here, superadditive functions are basic to mixed integer programming as the next Proposition indicates.

Proposition 4. Johnson (1973); Jeroslow (1979)

If $X^{M I P}=\left\{(x, y) \in \mathbb{R}_{+}^{n_{1}^{1}} \times \mathbb{Z}_{+}^{n_{1}^{2}}: A_{1} x+A_{2} y \leq b\right\}, F: \mathbb{R}^{m} \rightarrow \mathbb{R}^{1}$ is superadditive and nondecreasing, and $\bar{F}$ exists, then

$$
\sum_{j=1}^{n_{1}^{1}} \bar{F}\left(a_{1 j}\right) x_{j}+\sum_{j=1}^{n_{1}^{2}} F\left(a_{2 j}\right) y_{j} \leq F(b)
$$

is a valid inequality for $X^{M I P}$, where $a_{1 j}$ and $a_{2 j}$ are the columns of $A_{1}$ and $A_{2}$ respectively.

Now we return to the lifting problem. When the function $\phi^{1}$ is superadditive on some appropriate cone $D$, the computation of the functions $\phi^{2}, \cdots, \phi^{K}$ can be avoided.

Proposition 5. If $\phi^{1}$ is superadditive on $D$, and $A^{2} t \in D$ for all $t \in X^{2}, \phi^{2}=\phi^{1}$ on $D$.

Proof. For $t \in X^{2}$ and $u \in D$,

$$
\begin{gathered}
\phi^{1}\left(u+A^{2} t\right)-\pi^{2} t \\
\geq \phi^{1}\left(u+A^{2} t\right)-\phi^{1}\left(A^{2} t\right) \text { as } \pi^{2} t \leq \phi^{1}\left(A^{2} t\right) \\
\geq \phi^{1}(u) \text { by superadditivity. }
\end{gathered}
$$

On the other hand as $0 \in X^{2}, \min _{t \in X^{2}}\left[\phi^{1}\left(u+A^{2} t\right)-\pi^{2} t\right] \leq \phi^{1}(u)$. Therefore for $u \in D, \phi^{2}(u)=\min _{t \in X^{2}}\left[\phi^{1}\left(u+A^{2} t\right)-\pi^{2} t\right]=\phi^{1}(u)$. 
Any function $\hat{\phi} \leq \phi^{1}$ is called a valid lifting fuction for $X^{2}$ because $\pi^{2} t \leq$ $\hat{\phi}\left(A^{2} t\right)$ for all $t \in X^{2}$ implies that $\pi^{1} z^{1}+\pi^{2} z^{2} \leq \pi_{0}+\nu s$ is valid for $Z^{2}(b)$. We say that $\hat{\phi}$ is used for lifting if $\pi^{2}$ satisfies $\pi^{2} t \leq \hat{\phi}\left(A^{2} t\right)$ for all $t \in X^{2}$. When in addition $\hat{\phi}$ is superadditive, more can be said.

Proposition 6. Suppose that $\hat{\phi} \leq \phi^{1}$ on $D$ and $\hat{\phi}$ is superadditive on $D$. If $A^{2} t \in$ $D$ for all $t \in X^{2}$ and $\hat{\phi}$ is used for lifting, then $\phi^{1} \geq \phi^{2} \geq \hat{\phi}$ on $D$.

Proof. As $\hat{\phi}$ is used for lifting, $\pi^{2} t \leq \hat{\phi}\left(A^{2} t\right)$ for $t \in X^{2}$. Now for $t \in X^{2}$ and $u \in D, \phi^{1}\left(u+A^{2} t\right)-\pi^{2} t \geq \phi^{1}\left(u+A^{2} t\right)-\hat{\phi}\left(A^{2} t\right) \geq \hat{\phi}\left(u+A^{2} t\right)-\hat{\phi}\left(A^{2} t\right) \geq \hat{\phi}(u)$ where the last two inequalities follow from $\hat{\phi} \leq \phi^{1}$ and the superadditivity of $\hat{\phi}$ on $D$ respectively. Thus $\phi^{2}(u)=\min _{t \in X^{2}}\left[\phi^{1}\left(u+A^{2} t\right)-\pi^{2} t\right] \geq \hat{\phi}(u)$ for $u \in D$. .

So $\hat{\phi}$ remains a valid lifting function for $\phi^{2}, \ldots, \phi^{K}$. Thus if such a superadditive function $\hat{\phi}$ is used for lifting, the ordering of the sets $X^{2}, \ldots, X^{K}$ and of the calculations is irrelevant, as shown by the following result.

Corollary 1. If $\hat{\phi} \leq \phi^{1}$ and $\hat{\phi}$ is superadditive on $D, \hat{\pi}^{k} t \leq \hat{\phi}\left(A^{k} t\right)$ and $A^{k} t \in D$ for all $t \in X^{k}$ and $k=2, \ldots, K$, then

$$
\pi^{1} z^{1}+\sum_{k=2}^{K} \hat{\pi}^{k} z^{k} \leq \pi_{0}+\nu s
$$

is valid for $Z^{K}(b)$.

Proof. If $\left(z^{1}, \ldots, z^{K}, s\right) \in Z^{K}(b), \pi^{1} z^{1}+\sum_{k=2}^{K} \hat{\pi}^{k} z^{k}$

$\leq \pi_{0}+\nu s-\phi^{1}\left(\sum_{k=2}^{K} A^{k} z^{k}\right)+\sum_{k=2}^{K} \hat{\phi}\left(A^{k} z^{k}\right)$

$\leq \pi_{0}+\nu s-\phi^{1}\left(\sum_{k=2}^{K} A^{k} z^{k}\right)+\hat{\phi}\left(\sum_{k=2}^{K} A^{k} z^{k}\right)$ using superadditivity $\leq \pi_{0}+\nu s$ as $\hat{\phi} \leq \phi^{1}$.

It is natural to ask whether functions such as $\hat{\phi}$ always exist.

Proposition 7. $\phi^{*}(u)=\min _{v \in D}\left[\phi^{1}(u+v)-\phi^{1}(v)\right]$ is superadditive on $D$, and $\phi^{*} \leq \phi^{1}$ on $D$.

Proof. For $u, v \in D, u+v \in D$ and so, for some $w \in D$,

$\phi^{*}(u+v)=\phi^{1}(u+v+w)-\phi^{1}(w)=\phi^{1}(u+v+w)-\phi^{1}(v+w)+\phi^{1}(v+w)-\phi^{1}(w)$ $\geq \phi^{*}(u)+\phi^{*}(v)$. Also $\phi^{*}(u) \leq \phi^{1}(u+\underline{0})-\phi(\underline{0})=\phi^{1}(u)$ for all $u \in D$.

If a superadditive function has been used to generate the initial valid inequality $\pi^{1} z^{1} \leq \pi_{0}+\nu s$ for $Z^{1}(b)$, there is a natural candidate to be used as a valid lifting function. 
Proposition 8. Suppose that the initial valid inequality for $Z^{1}(b)$ is of the form

$$
\sum_{j=1}^{n_{1}^{1}} \bar{F}\left(a_{1 j}\right) x_{j}^{1}+\sum_{j=1}^{n_{1}^{2}} F\left(a_{2 j}\right) y_{j}^{1} \leq F(b)
$$

with $F$ superadditive and nondecreasing on $\mathbb{R}^{m}$. Then $\hat{F}(u) \equiv F(b)-F(b-u)$ is a valid lifting function with $\hat{F}(u) \leq \phi^{1}(u)$ for all $u \in \mathbb{R}^{m}$.

Proof. $\phi^{1}(u)=F(b)-\max \left\{\sum_{j=1}^{n_{1}^{1}} \bar{F}\left(a_{1 j}\right) x_{j}^{1}+\sum_{j=1}^{n_{1}^{2}} F\left(a_{2 j}\right) y_{j}^{1}: A_{1}^{1} x^{1}+\right.$ $\left.A_{2}^{1} y^{1} \leq b-u,\left(x^{1}, y^{1}\right) \in \mathbb{R}^{n_{1}^{1}} \times \mathbb{Z}^{n_{1}^{2}}\right\} \geq F(b)-F(b-u)$ as $\sum_{j=1}^{n_{1}^{1}} \bar{F}\left(a_{1 j}\right) x_{j}^{1}+$ $\sum_{j=1}^{n_{1}^{2}} F\left(a_{2 j}\right) y_{j}^{1} \leq F(b-u)$ is a valid inequality for $Z^{1}(b-u)$.

Now suppose that the MIR function $F_{\alpha}$ is used to generate the first inequality.

Remark 7. If $f_{b}=\alpha, \hat{F}(u)=F_{\alpha}(b)-F_{\alpha}(b-u)=F_{\alpha}(u)$ for all $u \in \mathbb{R}^{m}$.

Thus $F_{\alpha}$ is itself a valid superadditive lifting function.

Example 4. (Simultaneous Lifting)

Consider the initial set $X$

$$
\begin{gathered}
5 y_{1}+5 y_{2}+5 y_{3}+x_{4}-x_{5} \leq 4+s \\
0 \leq x_{4} \leq 6 y_{4}, 0 \leq x_{5} \leq 8 y_{5} \\
y \in\{0,1\}^{5}, s \in \mathbb{R}_{+}^{1} .
\end{gathered}
$$

Setting $x_{4}=0, y_{4}=0, x_{5}=8, y_{5}=1$, we obtain the set $Y$

$$
\begin{gathered}
5 y_{1}+5 y_{2}+5 y_{3} \leq 12+s \\
y \in\{0,1\}^{3}, s \in \mathbb{R}_{+}^{1}
\end{gathered}
$$

of Example 3.

We now rewrite the set $X$ in the form (1).

$$
\begin{array}{ccc}
5 y_{1}+5 y_{2}+5 y_{3} \quad+x_{4}+ & +\bar{x}_{5}-s \\
\left(y_{1}, \ldots, y_{3}\right) \in X^{1},\left(x_{4}, y_{4}\right) \in X^{2},\left(\bar{x}_{5}, \bar{y}_{5}\right) \in X^{3}, s \in \mathbb{R}_{+}^{1}, & \leq 12
\end{array}
$$

where $\bar{x}_{5}=8-x_{5}, \bar{y}_{5}=1-y_{5}$,

$X^{1}=\{0,1\}^{3}, A^{1}=(5,5,5)$

$X^{2}=\left\{\left(x_{4}, y_{4}\right) \in \mathbb{R}_{+}^{1} \times\{0,1\}: x_{4} \leq 6 y_{4}\right\}, A^{2}=(1)$

$X^{3}=\left\{\left(\bar{x}_{5}, \bar{y}_{5}\right) \in \mathbb{R}^{1} \times\{0,1\}: 8 \geq \bar{x}_{5} \geq 8 \bar{y}_{5}\right\}, A^{3}=(1)$ and $b=12$.

Taking the same valid inequality as in Example 3, it is easily checked that its lifting function $\phi^{1}$ is superaditive on $\mathbb{R}_{+}^{1}$. As $A^{2} t \in \mathbb{R}_{+}^{1}$ for all $t \in X^{2}$ and $A^{3} t \in \mathbb{R}_{+}^{1}$ for all $t \in X^{3}$, Corollary 1 of Proposition 6 is applicable. 
For the set $X^{2}$, we have

$$
\Pi^{2}=\left\{(\lambda, \mu): \lambda x_{4}+\mu y_{4} \leq \phi^{1}\left(x_{4}\right) \text { for }\left(x_{4}, y_{4}\right) \in X^{2}\right\},
$$

and, as shown in Gu et al. (1999), we obtain valid lifting coefficients by taking a support to the lifting function $\phi^{1}$ over the range $[0,6]$. There are two extreme solutions $(\lambda, \mu)=(0,0)$ and $(\lambda, \mu)=\left(\frac{3}{4},-\frac{3}{2}\right)$.

For the set $X^{3}$,

$$
\Pi^{3}=\left\{(\lambda, \mu): \lambda \bar{x}_{5}+\mu \bar{y}_{5} \leq \phi\left(\bar{x}_{5}\right) \text { for }\left(\bar{x}_{5}, \bar{y}_{5}\right) \in X^{3}\right\}
$$

with unique extreme point $(\lambda, \mu)=(0, \phi(8))=(0,4)$.

So simultaneously lifting on the sets $X^{2}$ and $X^{3}$ gives the valid inequalities

$$
\begin{gathered}
3 y_{1}+3 y_{2}+3 y_{3}+4\left(1-y_{5}\right) \leq 6+s \text { and } \\
3 y_{1}+3 y_{2}+3 y_{3}+\left(\frac{3}{4} x_{4}-\frac{3}{2} y_{4}\right)+4\left(1-y_{5}\right) \leq 6+s .
\end{gathered}
$$

\subsection{Further Remarks on Lifting}

The Role of the Continuous Variables $s$.

The inclusion of the continuous variables $s$ in the description (1) of $Z^{k}(b)$ for all $k=1, \ldots, K$ simplifies the presentation, but clearly restricts the inequalities that can be obtained by lifting. If the variables $s$ are set to zero and lifted later, we no longer have that $Z^{k}(b-u) \neq \emptyset$ for all $u$, with the result that $\phi^{k}$ can be discontinuous, and is not defined everywhere. Calculating $\phi^{k}$ and new coefficients $\pi^{k+1}$, and finding a valid superadditive function that is superadditive remain difficult problems. The resulting lifting functions $\phi^{k}$ are potentially stronger, but the final inequality may not be valid until the variables $s$ are lifted in. Examples of such functions can be found in Gu et al. (1999) among others.

\section{Facet-defining Inequalities.}

We have not discussed at all the question when the lifted inequalities are facetdefining. The brief answer is that if the set $Z^{1}(b)$ is full-dimensional, the initial inequality is facet-defining, the exact lifting function is used to define $\Pi^{k}$ and $\pi^{k}$ is an extreme point of $\Pi^{k}$ for all $k$, then the final inequality (2) is facet-defining for $Z^{K}(b)$. When the sets are not full-dimensional, more conditions are needed. See Oosten (1996) for a detailed study of this question. Note also that most of the papers cited in the Introduction present conditions under which the inequalities derived are facet-defining. 


\section{0-1 Single Node Flow Sets}

As indicated in the Introduction, single node flow sets $X^{N}$ are a natural generalization of knapsack sets and single row mixed integer sets. As suggested in section 2, we can take two approaches. The first: studying $\operatorname{conv}\left(X^{N}\right)$ in depth and thereby obtaining complete information about the special cases $Y$ (the 0-1 knapsack set with a continuous variable $X^{C K}$, or the single node flow with simple bounds $X^{N C}$ ) is unfortunately still an important challenge. The second, using knowledge about $Y=X^{C K}$ to obtain important, but partial knowledge about the superset $X^{N}$, is pursued here. The presentation is related to that of Marchand and Wolsey (2001). Surprisingly we show that by using mixed integer rounding (combined with superadditive lifting), one obtains inequalities as least as strong as all the flow cover inequalities for $X^{N}$ proposed earlier.

\subsection{The MIR approach}

From now on we will use a standard approach to generate valid inequalities for the single node flow set $X^{N}$ and its variants, which is a minor modification of the $c-M I R$ approach in Marchand and Wolsey (1999).

Step 1 Using slack variables for the variable upper bound constraints, relax $X^{N}$ to obtain a knapsack set with a continuous variable $X^{K C}$.

Step $1 \mathbf{b}$ (Optional) Fix the values of some variables giving a restricted set $X^{K C-F}$. Step 2 Complement certain integer variables - those in an appropriately chosen "cover".

Step 3 Rescale the row.

Step 4 Generate a mixed integer rounding inequality for $X^{K C}$ or $X^{K C-F}$, and rescale the inequality.

Step $4 \mathbf{b}$ If variables have been fixed in Step 1b, calculate the lifting function $\phi^{1}$. If the lifting function is not superadditive on some appropriate domain, look for a valid superadditive lifting function $\hat{\phi}$. Generate a valid inequality for $X^{K C}$.

Step 5 By complementing again, and eliminating the slack variables introduced in Step 1, generate a valid inequality for $X^{N}$.

\subsection{The MIR Flow Cover Inequalities}

Consider the set $X^{N}\left(n_{1}, n_{2}, b, a, \underline{1}\right)$. We now use the MIR approach described above to derive basic valid inequalities for this set.

Definition 5. $\left(C_{1}, C_{2}\right)$ is a flow cover for $X^{N}$ if

i) $C_{1} \subseteq N_{1}, C_{2} \subseteq N_{2}$

ii) $\sum_{j \in C_{1}} a_{j}-\sum_{j \in C_{2}} a_{j}-b=\lambda>0$. 
Proposition 9. Suppose that $\left(C_{1}, C_{2}\right)$ is a flow cover, and choose $\bar{a} \in \mathbb{R}_{+}^{1}$ with $\bar{a}>\lambda$. Then the MIR flow cover inequality

$$
\begin{array}{r}
\sum_{j \in C_{1}}\left\{x_{j}+\left[a_{j}+\lambda F\left(-\frac{a_{j}}{\bar{a}}\right)\right]\left(1-y_{j}\right)\right\} \\
+\sum_{j \in L_{1}} x_{j}-\sum_{j \in L_{1}}\left[a_{j}-\lambda F\left(\frac{a_{j}}{\bar{a}}\right)\right] y_{j} \\
\leq b+\sum_{j \in C_{2}} a_{j}-\sum_{j \in C_{2}} \lambda F\left(\frac{a_{j}}{\bar{a}}\right)\left(1-y_{j}\right) \\
-\sum_{j \in L_{2}} \lambda F\left(-\frac{a_{j}}{\bar{a}}\right) y_{j}+\sum_{j \in R_{2}} x_{j}+s
\end{array}
$$

is valid for $X^{N}$, where $\left(C_{i}, L_{i}, R_{i}\right)$ is a partition of $N_{i}$ for $i=1,2$ and $F=F_{\alpha}$ with $\alpha=\frac{\bar{a}-\lambda}{\bar{a}}$.

Proof. Step 1 Starting from the inequality

$$
\sum_{j \in N_{1}} x_{j}-\sum_{j \in N_{2}} x_{j} \leq b+s
$$

we introduce variables $t_{j}=a_{j} y_{j}-x_{j}$ for $j \in C_{1} \cup L_{1} \cup C_{2} \cup L_{2}$. Using the nonnegativity of $x_{j}$ for $j \in R_{1}$ and of $t_{j}$ for $j \in C_{2} \cup L_{2}$ gives the relaxation

$$
\sum_{j \in C_{1} \cup L_{1}} a_{j} y_{j}-\sum_{j \in C_{2} \cup L_{2}} a_{j} y_{j}-\sum_{j \in R_{2}} x_{j} \leq b+\sum_{j \in C_{1} \cup L_{1}} t_{j}+s .
$$

Step 2 Now introducing variables $\bar{y}_{j}=1-y_{j}$ for $j \in C_{1} \cup C_{2}$, we obtain

$-\sum_{j \in C_{1}} a_{j} \bar{y}_{j}+\sum_{j \in L_{1}} a_{j} y_{j}+\sum_{j \in C_{2}} a_{j} \bar{y}_{j}-\sum_{j \in L_{2}} a_{j} y_{j} \leq-\lambda+\sum_{j \in R_{2}} x_{j}+\sum_{j \in C_{1} \cup L_{1}} t_{j}+s$.

Step 3 We now divide by $\bar{a}>\lambda$.

Step 4 Generate the mixed integer rounding inequality giving

$$
\begin{array}{r}
\sum_{j \in C_{1}} F\left(-\frac{a_{j}}{\bar{a}}\right) \bar{y}_{j}+\sum_{j \in L_{1}} F\left(\frac{a_{j}}{\bar{a}}\right) y_{j}+\sum_{j \in C_{2}} F\left(\frac{a_{j}}{\bar{a}}\right) \bar{y}_{j}+\sum_{j \in L_{2}} F\left(-\frac{a_{j}}{\bar{a}}\right) y_{j} \\
\leq-1+\frac{1}{\lambda}\left(s+\sum_{j \in R_{2}} x_{j}+\sum_{j \in C_{1} \cup L_{1}} t_{j}\right) .
\end{array}
$$

Step 5 Multiplying by $\lambda$, and restating the inequality in terms of the original variables gives the required inequality.

Remark 8. i) $\lambda F\left(-\frac{a_{j}}{\bar{a}}\right) \geq-\max \left[\min \left[\lambda, a_{j}\right], a_{j}-(\bar{a}-\lambda)\right]$ with equality for $a_{j} \leq$ $\bar{a}+\lambda$.

ii) $\lambda F\left(-\frac{a_{j}}{\bar{a}}\right)=-\min \left[\lambda, a_{j}\right]$ for $a_{j} \leq \bar{a}$.

iii) $\lambda F\left(\frac{a_{j}}{\bar{a}}\right) \geq \min \left[\max \left[\left(a_{j}-(\bar{a}-\lambda), 0\right], \lambda\right]\right.$ with equality for $a_{j} \leq 2 \bar{a}-\lambda$.

iv) $\lambda F\left(\frac{a_{j}}{\bar{a}}\right)=\max \left[\left(a_{j}-(\bar{a}-\lambda), 0\right]\right.$ if $a_{j} \leq \bar{a}$. 
Corollary 2. If $\bar{a}=\max _{j \in C_{1}} a_{j}$, the MIR flow cover inequality (3) takes the form

$$
\begin{gathered}
\sum_{j \in C_{1}}\left\{x_{j}+\left[a_{j}-\lambda\right]^{+}\left(1-y_{j}\right)\right\}+\sum_{j \in L_{1}} x_{j}-\sum_{j \in L_{1}}\left[a_{j}-\lambda F\left(\frac{a_{j}}{\bar{a}}\right)\right] y_{j} \\
\leq b+\sum_{j \in C_{2}} a_{j}-\sum_{j \in C_{2}} \lambda F\left(\frac{a_{j}}{\bar{a}}\right)\left(1-y_{j}\right)-\sum_{j \in L_{2}} \lambda F\left(-\frac{a_{j}}{\bar{a}}\right) y_{j}+\sum_{j \in R_{2}} x_{j}+s
\end{gathered}
$$

and is at least as strong as the GFC2 inequality (Van Roy and Wolsey, 1986)

$$
\begin{array}{r}
\sum_{j \in C_{1}} x_{j}+\sum_{j \in C_{1}}\left[a_{j}-\lambda\right]^{+}\left(1-y_{j}\right)+\sum_{j \in L_{1}} x_{j}-\sum_{j \in L_{1}}\left(\max \left[a_{j}, \bar{a}\right]-\lambda\right) y_{j} \\
\leq b+\sum_{j \in C_{2}} a_{j}-\sum_{j \in C_{2}} \min \left[\lambda,\left(a_{j}-(\bar{a}-\lambda)\right)^{+}\right]\left(1-y_{j}\right) \\
+\sum_{j \in L_{2}} \max \left[a_{j}-(\bar{a}-\lambda), \lambda\right] y_{j}+\sum_{j \in R_{2}} x_{j}+s .
\end{array}
$$

Corollary 3. If $\bar{a}=\max _{j \in C_{1} \cap L_{2}} a_{j}$ and $a_{j}>\lambda$ for all $j \in L_{2}$, the MIR flow cover inequality (3) takes the form

$$
\begin{aligned}
& \sum_{j \in C_{1}}\left\{x_{j}+\left[a_{j}-\lambda\right]^{+}\left(1-y_{j}\right)\right\}+\sum_{j \in L_{1}} x_{j}-\sum_{j \in L_{1}}\left[a_{j}-\lambda F\left(\frac{a_{j}}{\bar{a}}\right)\right] y_{j} \\
& \leq b+\sum_{j \in C_{2}} a_{j}-\sum_{j \in C_{2}} \lambda F\left(\frac{a_{j}}{\bar{a}}\right)\left(1-y_{j}\right)+\sum_{j \in L_{2}} \lambda y_{j}+\sum_{j \in R_{2}} x_{j}+s
\end{aligned}
$$

and is at least as strong as the GFC1 inequality (Van Roy and Wolsey, 1986)

$$
\sum_{j \in C_{1}}\left\{x_{j}+\left[a_{j}-\lambda\right]^{+}\left(1-y_{j}\right)\right\} \leq b+\sum_{j \in C_{2}} a_{j}+\sum_{j \in L_{2}} \lambda y_{j}+\sum_{j \in R_{2}} x_{j}+s .
$$

\subsection{A Strengthened MIR Flow Cover Inequality}

Now we strengthen the inequality.

Proposition 10. Suppose that $\left(C_{1}, C_{2}\right)$ is a flow cover and $\bar{a}=\max _{j \in C_{1} \cup L_{2}} a_{j}>$ $\lambda$. Then the lifted inequality

$$
\begin{array}{r}
\sum_{j \in C_{1}}\left\{x_{j}+\left[a_{j}-\lambda\right]^{+}\left(1-y_{j}\right)\right\} \\
+\sum_{j \in L_{1}}\left[x_{j}-\left(a_{j}-\phi^{1}\left(a_{j}\right)\right) y_{j}\right] \\
\leq b+\sum_{j \in C_{2}} a_{j}-\sum_{j \in C_{2}} \phi^{1}\left(a_{j}\right)\left(1-y_{j}\right) \\
-\sum_{j \in L_{2}} \lambda y_{j}+\sum_{j \in R_{2}} x_{j}+s
\end{array}
$$

is valid for $X^{N}$, where $\left(C_{i}, L_{i}, R_{i}\right)$ is a partition of $N_{i}$ for $i=1,2$ and $\phi^{1}=G_{a, \lambda}$ on $\mathbb{R}_{+}^{1}$ with $a=\left(a^{C_{1}}, a^{L_{2}}\right)$. 
Proof. Proceeding as in the proof of Proposition 9, we modify as follows:

Step 1b Set $y_{j}=0$ for $j \in L_{1}$ and $\bar{y}_{j}=0$ for $j \in C_{2}$.

Step 2 The restricted set takes the form:

$$
-\sum_{j \in C_{1}} a_{j} \bar{y}_{j}-\sum_{j \in L_{2}} a_{j} y_{j} \leq-\lambda+\sum_{j \in R_{2}} x_{j}+\sum_{j \in C_{1} \cup L_{1}} t_{j}+s .
$$

Step 3. Divide by $\bar{a}=\max _{j \in C_{1} \cup L_{2}} a_{j}$.

Step 4. Generate the MIR inequality

$$
-\sum_{j \in C_{1}} \min \left[\lambda, a_{j}\right] \bar{y}_{j}-\sum_{j \in L_{2}} \lambda y_{j} \leq-\lambda+\sigma
$$

where $\sigma=\sum_{j \in R_{2}} x_{j}+\sum_{j \in C_{1} \cup L_{1}} t_{j}+s$.

Step $4 \mathbf{b}$ Calculate the lifting function

$$
\begin{aligned}
& \phi^{1}(u)=\min \sum_{j \in C_{1}} \min \left[\lambda, a_{j}\right] \bar{y}_{j}+\sum_{j \in L_{2}} \lambda y_{j}-\lambda+\sigma, \\
&-\sum_{j \in C_{1}} a_{j} \bar{y}_{j}-\sum_{j \in L_{2}} a_{j} y_{j}-\sigma \leq-\lambda-u \\
& \bar{y}_{j} \in\{0,1\} \text { for } j \in C_{1}, y_{j} \in\{0,1\} \text { for } j \in L_{2}, \sigma \geq 0 .
\end{aligned}
$$

It can be shown that on $\mathbb{R}_{+}^{1}, \phi^{1}$ is precisely the superadditive function $G_{a, \lambda}$ with $a=\left(a^{C_{1}}, a^{L_{2}}\right)$.

Lift to obtain the inequality

$$
-\sum_{j \in C_{1}} \min \left[\lambda, a_{j}\right] \bar{y}_{j}-\sum_{j \in L_{2}} \lambda y_{j}+\sum_{j \in L_{1}} G_{a, \lambda}\left(a_{j}\right) y_{j}+\sum_{j \in C_{2}} G_{a, \lambda}\left(a_{j}\right) \bar{y}_{j} \leq-\lambda+\sigma .
$$

Step 5. Uncomplement variables and substitute for $t_{j}$.

Example 5. Consider the single node flow set

$$
\begin{gathered}
x_{1}+x_{2}-x_{3}+x_{4}+x_{5}-x_{6} \leq-8+s \\
x_{1} \leq 10 y_{1}, x_{2} \leq 9 y_{2}, x_{3} \leq 7 y_{3}, x_{4} \leq 16 y_{4}, x_{5} \leq 5 y_{5}, x_{6} \leq 19 y_{6} \\
x \in \mathbb{R}_{+}^{6}, s \in \mathbb{R}_{+}^{1}, y \in[0,1]^{6} .
\end{gathered}
$$

Taking as flow cover $C_{1}=\{1,2\}, C_{2}=\{6\}$, we obtain $\lambda=10+9-19+8=8$. With $L_{1}=\{4\}, L_{2}=\emptyset$, we take $\bar{a}=\max _{j \in C_{1} \cup L_{2}} a_{j}=10, \alpha=\frac{10-8}{10}$.

The resulting MIR flow cover inequality (4) is

$$
x_{1}+2\left(1-y_{1}\right)+x_{2}+1\left(1-y_{2}\right)-x_{3}+x_{4}-4 y_{4} \leq 11-15\left(1-y_{6}\right)+s .
$$

To obtain the strengthened inequality (5), we calculate the lifting function $\phi^{1}$. With $a=(10,9), \lambda=8$ 


$$
G_{a, \lambda}(u)=\left\{\begin{array}{c}
0 \quad \text { if } 0 \leq u \leq 2 \\
u-2 \text { if } 2 \leq u \leq 10 \\
8 \text { if } 10 \leq u \leq 11 \\
u-3 \text { if } 11 \leq u
\end{array}\right.
$$

As $G_{a, \lambda}(16)=13$ and $G_{a, \lambda}(19)=16$, we obtain the inequality

$x_{1}+2\left(1-y_{1}\right)+x_{2}+1\left(1-y_{2}\right)-x_{3}+x_{4}-(16-13) y_{4} \leq 11-16\left(1-y_{6}\right)+s$

which in this case is stronger than the MIR inequality.

\subsection{The MIR Reverse Flow Cover Inequality}

We now present an explicit expression for the reverse flow cover inequality for this set. This inequality is obtained by applying the results of the previous subsection to the single node flow set in which the directions of the flows are all reversed.

Definition 6. $\left(T_{1}, T_{2}\right)$ is a reverse flow cover for $X^{N}$ if

i) $T_{1} \subseteq N_{1}, T_{2} \subseteq N_{2}$

ii) $\sum_{j \in T_{1}} a_{j}-\sum_{j \in T_{2}} a_{j}-b=-\mu<0$.

Proposition 11. Suppose that $\left(T_{1}, T_{2}\right)$ is a reverse flow cover and $\bar{a}>\mu$. Then the MIR reverse flow cover inequality

$$
\begin{array}{r}
\left.\sum_{j \in T_{1}} x_{j}+\sum_{j \in T_{1}} \mu F\left(\frac{a_{j}}{\bar{a}}\right)\right]\left(1-y_{j}\right) \\
\left.+\sum_{j \in L_{1}} x_{j}+\sum_{j \in L_{1}} \mu F\left(-\frac{a_{j}}{\bar{a}}\right)\right] y_{j} \\
\leq \sum_{j \in T_{1}} a_{j}-\sum_{j \in T_{2}}\left[a_{j}+\mu F\left(-\frac{a_{j}}{\bar{a}}\right)\right]\left(1-y_{j}\right) \\
+\sum_{j \in L_{2}}\left[a_{j}-\mu F\left(\frac{a_{j}}{\bar{a}}\right)\right] y_{j}+\sum_{j \in R_{2}} x_{j}+s .
\end{array}
$$

is valid for $X^{N}$, where $\left(T_{i}, L_{i}, R_{i}\right)$ is a partition of $N_{i}$ for $i=1,2$ and $F=F_{\alpha}$ with $\alpha=\frac{\bar{a}-\mu}{\bar{a}}$.

Corollary 4. If $\bar{a}=\max _{j \in T_{2}} a_{j}$, the MIR reverse flow cover takes the form

$$
\begin{array}{r}
\left.\sum_{j \in T_{1}} x_{j}+\sum_{j \in T_{1}} \mu F\left(\frac{a_{j}}{\bar{a}}\right)\left(1-y_{j}\right)+\sum_{j \in L_{1}} x_{j}+\sum_{j \in L_{1}} \mu F\left(-\frac{a_{j}}{\bar{a}}\right)\right] y_{j} \\
\leq \sum_{j \in T_{1}} a_{j}-\sum_{j \in T_{2}}\left(a_{j}-\mu\right)^{+}\left(1-y_{j}\right)+\sum_{j \in L_{2}}\left[a_{j}-\mu F\left(\frac{a_{j}}{\bar{a}}\right)\right] y_{j}+\sum_{j \in R_{2}} x_{j}+s,
\end{array}
$$

and is at least as strong as the inequality 


$$
\begin{array}{r}
\sum_{j \in T_{1}} x_{j}+\sum_{j \in T_{1}} \min \left[a_{j}-(\bar{a}-\mu)^{+}, \mu\right]\left(1-y_{j}\right) \\
+\sum_{j \in L_{1}} x_{j}-\sum_{j \in L_{1}} \max \left[\min \left(\mu, a_{j}\right), a_{j}-(\bar{a}-\mu)\right] y_{j} \\
\leq \sum_{j \in T_{1}} a_{j}-\sum_{j \in T_{2}}\left(a_{j}-\mu\right)^{+}\left(1-y_{j}\right) \\
+\sum_{j \in L_{2}}\left[\max \left(a_{j}-\mu, \min \left\{a_{j}, \bar{a}-\mu\right\}\right] y_{j}+\sum_{j \in R_{2}} x_{j}+s,\right.
\end{array}
$$

obtained by fixing the variable lower bounds to zero in the inequalities of Stallaert (1997).

Corollary 5. If $\bar{a}$ is large, the MIR reverse flow cover takes the form

$$
\sum_{j \in T_{1}} x_{j}+\sum_{j \in L_{1}}\left(x_{j}-\mu y_{j}\right) \leq \sum_{j \in T_{1}} a_{j}-\sum_{j \in T_{2}}\left(a_{j}-\mu\right)^{+}\left(1-y_{j}\right)+\sum_{j \in L_{2} \cup R_{2}} x_{j}+s .
$$

\subsection{Further Remarks}

The main difference between the MIR approach proposed here and the $c-M I R$ approach in Marchand and Wolsey (2001) is the role of the cover in determining which variables to complement. None of the inequalities proposed in this section is really new. In particular the strengthened MIR flow cover inequality is essentially derived in Marchand and Wolsey (1999) and can also be seen as a special case of the LSGFCI inequality in Gu et al. (1999) when there is an unbounded continuous variable. Also as remarked above, the reverse flow cover inequalities are nothing but flow cover inequalities, and arc reversal was already used in Van Roy and Wolsey (1987).

\section{Integer Single Node Flow Sets}

Here we consider the case where the integer variables arising in the single node flow set are bounded, but not all $0-1$. The flow cover inequality proposed below is apparently new. However the strengthening procedure is that for integer knapsack sets proposed by Atamtürk (2002).

\subsection{The MIR Flow Cover Inequality}

Consider now the set $X^{N}\left(n_{1}, n_{2}, b, a, u\right)$ with $u_{j}>1$ for some $j \in N$. We now derive an MIR flow cover inequality for this set. 
Definition 7. $\left(C_{1}, C_{2}\right)$ is an integer flow cover for $X^{N}$ if i) $C_{1} \subseteq N_{1}, C_{2} \subseteq N_{2}$

ii) there exists $k \in C_{1}$ such that $\sum_{j \in C_{1} \backslash k} a_{j} u_{j}-\sum_{j \in C_{2}} a_{j} u_{j}<b$ and there exists unique values $\lambda$ and $\eta_{k}$ such that

$$
a_{k} \eta_{k}+\sum_{j \in C_{1} \backslash k} a_{j} u_{j}-\sum_{j \in C_{2}} a_{j} u_{j}=b+\lambda
$$

with $0<\lambda<a_{k}$, and $\eta_{k} \in \mathbb{Z}^{1}$ with $1 \leq \eta_{k} \leq u_{k}$.

Proposition 12. Suppose that $\left(C_{1}, C_{2}\right)$ is an integer flow cover. Then the integer flow cover inequality

$$
\begin{array}{r}
\sum_{j \in C_{1}} x_{j}+\left(a_{k}-\lambda\right)\left(\eta_{k}-y_{k}\right)+\sum_{j \in C_{1} \backslash k}\left[a_{j}+\lambda F\left(-\frac{a_{j}}{a_{k}}\right)\right]\left(u_{j}-y_{j}\right) \\
+\sum_{j \in L_{1}} x_{j}-\sum_{j \in L_{1}}\left[a_{j}-\lambda F\left(\frac{a_{j}}{a_{k}}\right)\right] y_{j} \\
\leq b+\sum_{j \in C_{2}} a_{j} u_{j}-\sum_{j \in C_{2}} \lambda F\left(\frac{a_{j}}{a_{k}}\right)\left(u_{j}-y_{j}\right) \\
-\sum_{j \in L_{2}} \lambda F\left(-\frac{a_{j}}{a_{k}}\right) y_{j}+\sum_{j \in R_{2}} x_{j}+s
\end{array}
$$

is valid for $X^{N}$, where $\left(C_{i}, L_{i}, R_{i}\right)$ is a partition of $N_{i}$ for $i=1,2$ and $F=F_{\alpha}$ with $\alpha=\frac{a_{k}-\lambda}{a_{k}}$.

Proof. We again use the MIR approach from the previous section. Starting from the inequality

$$
\sum_{j \in N_{1}} x_{j}-\sum_{j \in N_{2}} x_{j} \leq b+s,
$$

we introduce variables $t_{j}=a_{j} y_{j}-x_{j}$ for $j \in C_{1} \cup L_{1} \cup C_{2} \cup L_{2}$. Using the nonnegativity of $x_{j}$ for $j \in R_{1}$ gives the relaxation

$$
\sum_{j \in C_{1} \cup L_{1}} a_{j} y_{j}-\sum_{j \in C_{2} \cup L_{2}} a_{j} y_{j}-\sum_{j \in R_{2}} x_{j} \leq b+\sum_{j \in C_{1} \cup L_{1}} t_{j}+s .
$$

Now introducing variables $\bar{y}_{j}=u_{j}-y_{j}$ for $j \in C_{1} \cup C_{2}$, we obtain

$$
\begin{gathered}
-\sum_{j \in C_{1}} a_{j} \bar{y}_{j}+\sum_{j \in L_{1}} a_{j} y_{j}+\sum_{j \in C_{2}} a_{j} \bar{y}_{j}-\sum_{j \in L_{2}} a_{j} y_{j} \\
\quad \leq a_{k}\left(\eta_{k}-u_{k}\right)-\lambda+\sum_{j \in R_{2}} x_{j}+\sum_{j \in C_{1} \cup L_{1}} t_{j}+s .
\end{gathered}
$$


We now divide by $a_{k}$, and then generate the mixed integer rounding inequality giving

$$
\begin{aligned}
& \sum_{j \in C_{1}} F\left(-\frac{a_{j}}{a_{k}}\right) \bar{y}_{j}+ \sum_{j \in L_{1}} F\left(\frac{a_{j}}{a_{k}}\right) y_{j}+\sum_{j \in C_{2}} F\left(\frac{a_{j}}{a_{k}}\right) \bar{y}_{j}+\sum_{j \in L_{2}} F\left(-\frac{a_{j}}{a_{k}}\right) y_{j} \\
& \leq\left(\eta_{k}-u_{k}\right)-1+\frac{1}{\lambda}\left(s+\sum_{j \in R_{2}} x_{j}+\sum_{j \in C_{1} \cup L_{1}} t_{j}\right) .
\end{aligned}
$$

Multiplying by $\lambda$, and restating the inequality in terms of the original variables gives the required inequality.

\subsection{Strengthening the Integer Flow Cover Inequality}

To obtain stronger inequalities, we use results of Atamtürk (2002) on integer knapsack sets. We start from inequality (8) in the proof of validity of Proposition 12 which we write more compactly, after recomplementing $\bar{y}_{k}$, as

$$
\begin{gathered}
a_{k} z_{k}-\sum_{j \in I^{-}} a_{j} z_{j}+\sum_{j \in I^{+}} a_{j} z_{j} \leq a_{k} \eta_{k}-\lambda+\sigma \\
z_{j} \leq u_{j}, z_{j} \in \mathbb{Z}_{+}^{1} \text { for } j \in\{k\} \cup I^{-} \cup I^{+}, \sigma \in \mathbb{R}_{+}^{1} .
\end{gathered}
$$

where $I^{-}=C_{1} \backslash\{k\} \cup L_{2}, I^{+}=C_{2} \cup L_{1}, \sigma=s+\sum_{j \in R_{2}} x_{j}+\sum_{j \in C_{1} \cup L_{1}} t_{j}$ and $z_{j}$ represents either $y_{j}$ or $\bar{y}_{j}$ as appropriate.

Setting $z_{j}=0$ for $j \in I^{-} \cup I^{+}$, leads to the reduced system

$$
\begin{gathered}
a_{k} z_{k}-\sigma \leq a_{k} \eta_{k}-\lambda \\
z_{k} \leq u_{k}, z_{k} \in \mathbb{Z}_{+}^{1}, \sigma \in \mathbb{R}_{+}^{1}
\end{gathered}
$$

with valid inequality

$$
\lambda z_{k} \leq \lambda\left(\eta_{k}-1\right)+\sigma .
$$

The lifting function for this inequality is easily calculated, is identical to $\lambda F_{\frac{a_{k}-\lambda}{a_{k}}}$ around the origin, and explicitly takes into account the upper and lower bounds on $z_{k}$ :

$$
\phi(v)=\left\{\begin{array}{cc}
\left(\eta_{k}-u_{k}-1\right) \lambda & \text { if } \quad v \leq a_{k}\left(\eta_{k}-u_{k}\right)-\lambda \\
(j-1) \lambda+\left[v-\left(j a_{k}-\lambda\right)\right] & \text { if } \quad j a_{k}-\lambda \leq v \leq j a_{k}, \\
j=\left(\eta_{k}-u_{k}\right), \ldots, \eta_{k}-1 \\
j \lambda & \text { if } \begin{array}{c}
j a_{k} \leq v \leq(j+1) a_{k}-\lambda, \\
j=\left(\eta_{k}-u_{k}\right), \ldots, \eta_{k}-1
\end{array} \\
& \eta_{k} a_{k}-\lambda \leq v .
\end{array}\right.
$$

This function is superadditive on $\mathbb{R}_{+}^{1}$ and separately on $\mathbb{R}_{-}^{1}$. 
Here we just consider the case where we first lift in the variables in $I^{-}$, and then the variables in $I^{+}$. Because $\phi$ is superadditive on $\mathbb{R}_{-}^{1}$, the lifting coefficients do not change as these variables in $I^{-}$are lifted in. After lifting in the variables in $I^{-}$, we obtain the lifting function $\phi^{+}$.

This function turns out to be superadditive on $\mathbb{R}_{+}^{1}$ under certain conditions, see Appendix. In the general case, we obtain a valid lifting function $\mathrm{H}^{+}$superadditive on $\mathbb{R}_{+}^{1}$ by dropping the nonnegativity constraint on $z_{k}$ in the mixed integer program defining $\phi^{+}$, namely

$$
\begin{array}{r}
H^{+}(v)=\min \left\{\lambda\left(\eta_{k}-1\right)-\lambda z_{k}+\sigma-\sum_{j \in I^{-}} \phi\left(-a_{j}\right) z_{j}:\right. \\
a_{k} z_{k}-\sum_{j \in I^{-}} a_{j} z_{j}-\sigma \leq a_{k} \eta_{k}-\lambda-v, \\
\left.z_{k} \leq u_{k}, z_{k} \in \mathbb{Z}^{1}, z_{j} \leq u_{j}, z_{j} \in \mathbb{Z}_{+}^{1} \text { for } j \in I^{-}, \sigma \in \mathbb{R}_{+}^{1} \cdot\right\}
\end{array}
$$

To describe $H^{+}$(and $\phi^{+}$), we define an ordering on the indices in $I^{-}, i_{1}, \cdots, i_{\mid I^{-}}$ such that $a_{i_{1}} \geq a_{i_{2}} \geq \cdots \geq a_{i_{\mid I^{-}} \mid}$. We also define the set $I^{--}=\{i: i \in$ $I^{-}$and $\left.a_{i} \geq a_{k}\left(u_{k}-\eta_{k}+1\right)\right\}$ with $\bar{r}=\max \left\{r: i_{r} \in I^{--}\right\}$. We also define $\rho_{r}=a_{k}\left(\eta_{k}-u_{k}\right)-\lambda+a_{i_{r}}$ for $r=1, \ldots, \bar{r}$. Finally, for an index $r \leq \bar{r}$, we consider two types of aggregate, $U_{r}=u_{i_{1}}+\cdots+u_{i_{r}}$, and $M_{r}=a_{i_{1}} u_{i_{1}}+\cdots+a_{i_{r}} u_{i_{r}}$.

It is not difficult to see that as $v$ increases from 0 , the mixed integer program defining $H^{+}(v)$ has optimal solutions in which the variables $i_{1}, \ldots, i_{r} \in I^{--}$are used in that order. Thus when $z_{i_{s}}=u_{i_{s}}$ for $s<r$ and $z_{i_{r}}=t, j=u_{k}-z_{k}$ takes values increasing from 0 to $u_{k}-\eta_{k}$. Once all of the variables in $I^{--}$are at their upper bound, $z_{k}$ then takes negative values. Specifically $H^{+}(v)=$

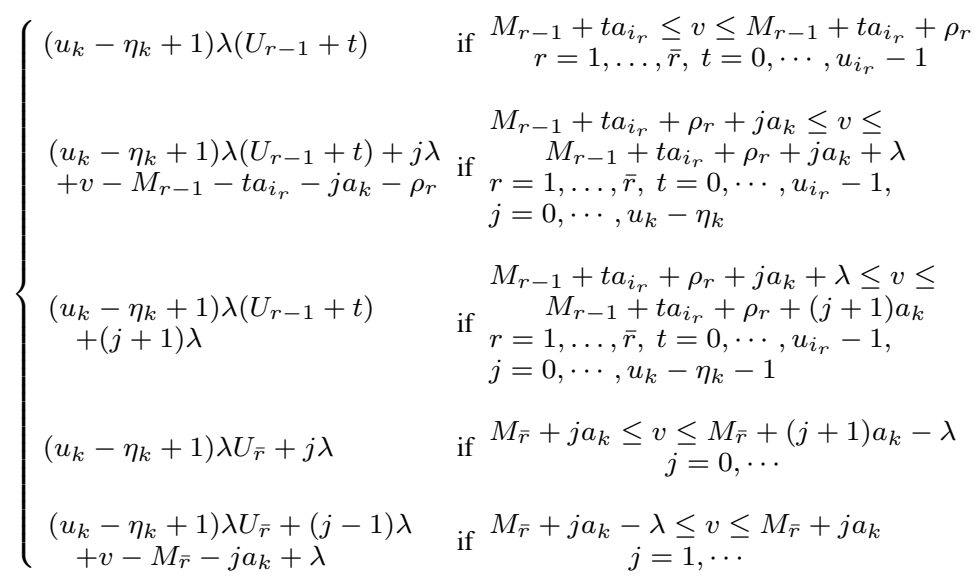

An example of the valid superadditive lifting function $H^{+}$and an exact lifting function $\phi^{+}$are depicted in Figure 5.

Finally $\mathrm{H}^{+}$can be used to lift in all the variables in $I^{+}$. 


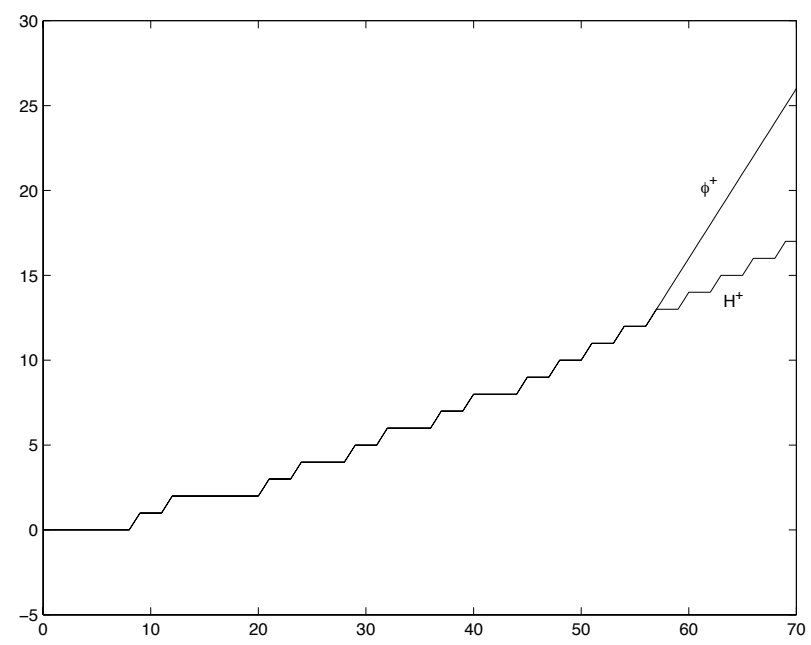

Fig. 5. Lifting functions $H^{+}$and $\phi^{+}$

Proposition 13. The inequality

$$
\begin{aligned}
& \lambda y_{k}+\sum_{j \in C_{1} \backslash\{k\}} \phi\left(-a_{j}\right)\left(1-y_{j}\right)+\sum_{j \in L_{2}} \phi\left(-a_{j}\right) y_{j} \\
& \quad+\sum_{j \in C_{2}} H^{+}\left(a_{j}\right)\left(1-y_{j}\right)+\sum_{j \in L_{1}} H^{+}\left(a_{j}\right) y_{j} \\
& \leq \lambda\left(\eta_{k}-1\right)+\sum_{j \in R_{2}} x_{j}+\sum_{j \in C_{1} \cup L_{1}}\left(a_{j} y_{j}-x_{j}\right)+s
\end{aligned}
$$

is valid for $X^{N}$.

In Atamtürk (2002), similar functions are also calculated for the case when one first lifts variables in $I^{+}$, and then those in $I^{-}$, which leads to another family of strong inequalities.

Example 6. Consider the set $X^{N}(2,2,4,(3,4,5,2),(2,3,2,3))$, namely

$$
\begin{gathered}
x_{1}+x_{2}-x_{3}-x_{4} \leq 4+s \\
x_{1} \leq 3 y_{1}, x_{2} \leq 4 y_{2}, x_{3} \leq 5 y_{3}, x_{4} \leq 2 y_{4} \\
y_{1} \leq 2, y_{2} \leq 3, y_{3} \leq 2, y_{4} \leq 3 \\
x \in \mathbb{R}_{+}^{4}, y \in \mathbb{Z}_{+}^{4}, s \in \mathbb{R}_{+}^{1} .
\end{gathered}
$$

Suppose that one wishes to cut off the fractional solution

$$
x^{*}=(2,12,10,0), y^{*}=\left(\frac{2}{3}, 3,2,0\right), s^{*}=0 .
$$

$C=\{1,2,3\}$ with $k=1$ is an integer flow cover with $a_{k}=3, \lambda=1$ and $\eta_{k}=1$. Inequality (7) with $L_{1}=L_{2}=\emptyset$ gives

$\left.x_{1}+(3-1)\left(1-y_{1}\right)+x_{2}+\left[4+F_{\frac{2}{3}}\left(-\frac{4}{3}\right)\right]\left(3-y_{2}\right) \leq 14-F_{\frac{2}{3}}\left(\frac{5}{3}\right)\right]\left(2-y_{3}\right)+x_{4}+s$, or 


$$
x_{1}+2\left(1-y_{1}\right)+x_{2}+2\left(3-y_{2}\right) \leq 14-1\left(2-y_{3}\right)+x_{4}+s
$$

which cuts off the fractional point, but is not facet-defining for $X^{N}$.

To try to obtain a stronger inequality, we again choose $C_{1}=\{1,2\}, C_{2}=\{3\}$ as a cover with $k=1$. Thus we consider

$$
3 y_{1}+4 y_{2}-5 y_{3}-x_{4} \leq 4+t_{1}+t_{2}+s \text {. }
$$

Complementing gives

$$
3 y_{1}-4 \bar{y}_{2}+5 \bar{y}_{3}-x_{4} \leq 2+t_{1}+t_{2}+s .
$$

Setting $\bar{y}_{2}=\bar{y}_{3}=0$ leaves the system

$$
3 y_{1}-x_{4} \leq 2+t_{1}+t_{2}+s
$$

with valid inequality

$$
y_{1}-x_{4} \leq t_{1}+t_{2}+s .
$$

Now the lifting function $\phi$ is given above. If we first lift in $\bar{y}_{2}, \phi(-4)=-2$ and we obtain

$$
y_{1}-2 \bar{y}_{2}-x_{4} \leq t_{1}+t_{2}+s .
$$

Using the superadditive function $H^{+}$to lift the variable $\bar{y}_{3}, H^{+}(5)=1$, so we obtain the same inequality as above.

If we calculate the exact lifting function, it turns out that $\phi^{+}(5)=2$ giving the inequality

$$
x_{1}-2 y_{1}-2 \bar{y}_{2}+2 \bar{y}_{3}-x_{4} \leq t_{2}+s,
$$

or

$$
x_{1}-2 y_{1}+x_{2}-2 y_{2}-2 y_{3}-x_{4} \leq 2+s
$$

which is facet-defining.

\subsection{The MIR Reverse Flow Cover Inequality}

Here we give an explicit formula for the reverse flow cover inequality when the bounds are integer. We modify ii) in Definition 7

Definition 8. $\left(T_{1}, T_{2}\right)$ is an integer reverse flow cover for $X^{N}$ if

i) $T_{1} \subseteq N_{1}, T_{2} \subseteq N_{2}$

ii) there exists $k \in T_{2}$ such that $\sum_{j \in T_{1}} a_{j} u_{j}-\sum_{j \in T_{2} \backslash k} a_{j} u_{j}>b$ and there exists unique values $\mu$ and $\eta_{k}$ such that

$$
\sum_{j \in T_{1}} a_{j} u_{j}-\sum_{j \in T_{2} \backslash k} a_{j} u_{j}-a_{k} \eta_{k}=b-\mu
$$

with $0<\mu<a_{k}$ and $\eta_{k} \in \mathbb{Z}^{1}$ with $1 \leq \eta_{k} \leq u_{k}$. 
Proposition 14. Suppose that $\left(T_{1}, T_{2}\right)$ is an integer reverse flow cover for $X^{N}$. The following inequality

$$
\begin{array}{r}
\sum_{j \in T_{1}}\left[x_{j}+\mu F\left(\frac{a_{j}}{a_{k}}\right)\left(u_{j}-y_{j}\right)\right]+\sum_{L_{1}}\left[x_{j}+\mu F\left(-\frac{a_{j}}{a_{k}}\right) y_{j}\right] \\
\leq \sum_{j \in T_{1}} a_{j} u_{j}-\left(a_{k}-\mu\right)\left(\eta_{k}-y_{k}\right)-\sum_{j \in T_{2} \backslash k}\left[a_{j}+\mu F\left(-\frac{a_{j}}{a_{k}}\right)\right]\left(u_{j}-y_{j}\right) \\
+\sum_{j \in L_{2}}\left[a_{j}-\mu F\left(\frac{a_{j}}{a_{k}}\right)\right] y_{j}+\sum_{j \in R_{2}} x_{j}+s
\end{array}
$$

is valid for $X^{N}$, where $\left(T_{i}, L_{i}, R_{i}\right)$ is a partition of $N_{i}$ for $i=1,2$ and $F=F_{\alpha}$ with $\alpha=\frac{a_{k}-\mu}{a_{k}}$.

Example 7. We consider the same set $X^{N}$ as in Example 6. Suppose that one wishes to cut off the fractional solution

$$
x^{*}=(0,12,2,6), y^{*}=\left(0,3, \frac{2}{5}, 2\right), s^{*}=0 .
$$

$T=\{2,3,4\}$ with $k=3$ is an integer reverse flow cover with $a_{k}=5, \eta_{k}=1$ and $\mu=3$. Inequality (9) with $L_{1}=L_{2}=\emptyset$ gives

$$
\begin{gathered}
x_{2}+3 F_{\frac{2}{5}}\left(\frac{4}{5}\right)\left(3-y_{2}\right) \leq 12-(5-3)\left(1-y_{3}\right)-\left(2+3 F_{\frac{2}{5}}\left(-\frac{2}{5}\right)\left(3-y_{4}\right)+s,\right. \text { or } \\
x_{2}+2\left(3-y_{2}\right) \leq 12-2\left(1-y_{3}\right)+s,
\end{gathered}
$$

which cuts off the fractional solution, and turns out to be facet-defining for $X^{N}$.

\section{Extensions}

Here we consider several extensions of the single node flow set. In particular we study a set with variable lower bounds of the form $l_{j} y_{j} \leq x_{j}$ which can also be related to problems with set-up times having flow constraints of the form $\sum_{j}\left(x_{j}+\right.$ $\left.b_{j} y_{j}\right) \leq b$, a set with generalized variable upper bounds of the form $x_{j} \leq a_{0 j}+$ $\sum_{i} a_{i j} y_{i j}$, and finally a set with generalized upper bounds $\sum_{i} y_{i j} \leq 1$.

\subsection{Variable Lower Bounds, or Set-up Times}

First we consider briefly the general case, namely the set

$$
\begin{gathered}
\sum_{j \in N_{1}} x_{j}-\sum_{j \in N_{2}} x_{j} \leq b+s \\
l_{j} y_{j} \leq x_{j} \leq a_{j} y_{j}, y_{j} \in\{0,1\} \text { for } j \in N_{1} \cup N_{2} .
\end{gathered}
$$


Valid inequalities for this model were first developed in Van Roy and Wolsey (1986), see also Marchand and Wolsey (1999); Stallaert (1997). To use the MIR approach, it suffices to choose as a cover disjoint sets $\left(C_{1}^{l}, C_{1}^{a}, C_{2}^{l}, C_{2}^{a}\right)$ for which

$$
\sum_{j \in C_{1}^{l}} l_{j}+\sum_{j \in C_{1}^{a}} a_{j}-\sum_{j \in C_{2}^{l}} l_{j}-\sum_{j \in C_{2}^{a}} a_{j}-b=\lambda>0
$$

Then given disjoint sets $L_{i}^{l}, L_{i}^{a} \subseteq N_{i} \backslash\left(C_{i}^{l} \cup C_{i}^{a}\right)$ for $i=1,2$, set $\bar{a}=$ $\max \left[\max _{j \in C_{1}^{l} \cup L_{2}^{l}} l_{j}, \max _{j \in C_{1}^{a} \cup L_{2}^{a}} a_{j}\right]$, introduce appropriate slacks, etc.

The resulting inequality

$$
\begin{array}{r}
\sum_{j \in C_{1}^{a}}\left\{x_{j}+\left(a_{j}-\lambda\right)^{+}\left(1-y_{j}\right)\right\}+\sum_{j \in C_{1}^{l}}\left\{\left[l_{j} y_{j}+\left(l_{j}-\lambda\right)^{+}\left(1-y_{j}\right)\right\}\right. \\
+\sum_{j \in L_{1}^{a}}\left[x_{j}-\left(a_{j}-\lambda F\left(\frac{a_{j}}{\bar{a}}\right) y_{j}\right]+\sum_{j \in L_{1}^{l}} \lambda F\left(\frac{l_{j}}{\bar{a}}\right) y_{j}\right. \\
\leq b+\sum_{j \in C_{2}^{a}} a_{j}+\sum_{j \in C_{2}^{l}} l_{j}-\sum_{j \in C_{2}^{a}} \lambda F\left(\frac{a_{j}}{\bar{a}}\right)\left(1-y_{j}\right) \\
+\sum_{j \in C_{2}^{l}}\left[x_{j}-l_{j} y_{j}-\lambda F\left(\frac{l_{j}}{\bar{a}}\right)\left(1-y_{j}\right)\right]+\sum_{j \in L_{2}^{a}} \lambda y_{j} \\
+\sum_{j \in L_{2}^{l}}\left[x_{j}-\left(l_{j}-\lambda\right)^{+} y_{j}\right]+\sum_{j \in R_{2}} x_{j}+s
\end{array}
$$

is valid for $X^{N}$, where $\left(C_{i}, L_{i}, R_{i}\right)$ is a partition of $N_{i}$ for $i=1,2$ and $F=F_{\alpha}$ with $\alpha=\frac{\bar{a}-\lambda}{\bar{a}}$.

We now consider the special case with $N_{2}=\emptyset$, namely the set $X^{V L B}$

$$
\begin{gathered}
\sum_{j \in N_{1}} x_{j} \leq b+s \\
l_{j} y_{j} \leq x_{j} \leq a_{j} y_{j}, y_{j} \in\{0,1\} \text { for } j \in N_{1} .
\end{gathered}
$$

where $l_{j} \geq 0$ for all $j \in N_{1}$.

Remark 9. By the change of variable $w_{j}=x_{j}-l_{j} y_{j} \geq 0$, this is equivalent to the "set-up time" model $X^{S T}$ :

$$
\begin{gathered}
\sum_{j \in N_{1}}\left(w_{j}+l_{j} y_{j}\right) \leq b+s \\
0 \leq w_{j} \leq \tilde{a}_{j} y_{j}, y_{j} \in\{0,1\} \text { for } j \in N_{1}, s \geq 0
\end{gathered}
$$

where $\tilde{a}_{j}=a_{j}-l_{j}$ for all $j \in N_{1}$. 
With $s$ fixed at zero, this set was first studied by Goemans (1989). Recently a closely related model has been studied by Miller et al. (2003a) in which $w_{j}$ represents the amount produced of item $j$ and $l_{j}$ is the set-up time for this item. We now present the latter model.

The set considered is

$$
\begin{gathered}
\sum_{j \in N_{1}}\left(w_{j}+l_{j} y_{j}\right) \leq b \\
w_{j} \leq \rho_{j} y_{j}+\sigma_{j}, w_{j} \leq M y_{j}, \text { for } j \in N_{1} \\
y \in\{0,1\}^{n_{1}}, w \in \mathbb{R}_{+}^{n_{1}}, \sigma \in \mathbb{R}_{+}^{n_{1}} .
\end{gathered}
$$

Making the change of variable $x_{j}=w_{j}+l_{j} y_{j} \leq\left(\rho_{j}+l_{j}\right) y_{j}+\sigma_{j}$, the GFC1 inequality (5) becomes

$$
\sum_{j \in C_{1}}\left(w_{j}+l_{j} y_{j}\right)+\sum_{j \in C_{1}}\left(\rho_{j}+l_{j}-\lambda\right)^{+}\left(1-y_{j}\right) \leq b+\sum_{j \in C_{1}} \sigma_{j}
$$

and the strengthened MIR inequality (5) becomes

$$
\begin{gathered}
\sum_{j \in C_{1}}\left(w_{j}+l_{j} y_{j}\right)+\sum_{j \in C_{1}}\left(\rho_{j}+l_{j}-\lambda\right)^{+}\left(1-y_{j}\right) \\
+\sum_{j \in L_{1}}\left[x_{j}-\rho_{j} y_{j}+G_{a, \lambda}\left(\rho_{j}+l_{j}\right) y_{j}\right] \leq b+\sum_{j \in C_{1} \cup L_{1}} \sigma_{j}
\end{gathered}
$$

where, in the definition of $G_{a, \lambda}, a_{j}=\rho_{j}+l_{j}$ for $j \in C_{1} \cup L_{2}$.

Example 8. The instance has $n=4, b=16, \rho=(5,4,5,10), l=(2,2,1,3)$.

Thus $a=\rho+l=(7,6,6,13)$.

Taking $C_{1}=\{1,2,3\}$ and $L_{1}=\{4\}, \lambda=3, \bar{a}=7$, and $G_{a, \lambda}(13)=6$ giving the inequality (11)

$$
\begin{array}{r}
w_{1}+2 y_{1}+(7-3)\left(1-y_{1}\right)+w_{2}+2 y_{2}+(6-3)\left(1-y_{2}\right) \\
+w_{3}+y_{3}+(6-3)\left(1-y_{3}\right)+x_{4}-(10-6) y_{4} \leq 16+\sum_{i=1}^{4} \sigma_{i}
\end{array}
$$

Substituting for $\sigma_{j}$ gives precisely the inequality (38) on p26 in Miller et al. (2003a).

\subsection{Generalized Variable Upper Bounds}

Here we consider the set $X^{G V U B}$

$$
\begin{gathered}
\sum_{j \in N_{1}} x_{j}-\sum_{j \in N_{2}} x_{j} \leq b+s \\
x_{j} \leq a_{0 j}+\sum_{i \in S_{j}} a_{i j} y_{i}, \text { for } j \in N . \\
x_{j} \geq 0 \text { for } j \in N, y_{i} \in\{0,1\} \text { for } i \in M .
\end{gathered}
$$

studied by Atamtürk et al. (2001). 
Remark 10. This set can be obtained by projecting a face of the single node flow model

$$
\begin{gathered}
\sum_{i, j} w_{i j}-\sum_{i, j} w_{i j} \leq b \\
0 \leq w_{i j} \leq a_{i j} y_{i j}, y_{i j} \in\{0,1\}
\end{gathered}
$$

by setting $y_{0 j}=1$ and $x_{j}=\sum_{i} w_{i j}$ for all $j$.

Here we again take a direct approach. Again we have a partition $\left(C_{k}, L_{k}, R_{k}\right)$ of $N_{k}$ for $k=1,2$ and a set $F \subseteq M$ of variables fixed at zero. For simplicity we assume that $L_{1}=\emptyset$ and $F \cap S_{j}=\emptyset$ for $j \in C_{2} \cup L_{2}$. To define a cover we need that

$$
\sum_{j \in C_{1}}\left[a_{0 j}+\sum_{i \in S_{j} \backslash F} a_{i j}\right]-\sum_{j \in C_{2}}\left[a_{0 j}+\sum_{i \in S_{j}} a_{i j}\right]-b-\sum_{j \in L_{2}} a_{0 j}=\lambda>0 .
$$

Assuming for simplicity that the sets $S_{j}$ are disjoint, we obtain using the standard MIR approach

Proposition 15. The inequality

$$
\begin{gathered}
\left.\sum_{j \in C_{1}}\left[x_{j}+\sum_{i \in S_{j} \backslash F}\left(a_{i j}-\lambda\right)^{+}\right)\left(1-y_{i}\right)\right]+\sum_{j \in C_{1}}\left[\sum_{i \in S_{j} \cap F} \phi\left(a_{i j}\right) y_{i}\right] \\
\leq b+\sum_{j \in C_{2}} \sum_{i \in S_{j}} a_{i j}+\sum_{j \in L_{2}} a_{0 j} \\
-\sum_{j \in C_{2}} \sum_{i \in S_{j}} \phi\left(a_{i j}\right)\left(1-y_{i}\right)+\sum_{j \in L_{2}} \sum_{i \in S_{j}} \min \left[\lambda, a_{i j}\right] y_{i}+\sum_{j \in R_{2}} x_{j}+s
\end{gathered}
$$

is valid for $X^{G V U B}$ where $\phi=G_{a, \lambda}$ and a consists of the terms $a_{i j}$ for $i \in S_{j} \backslash F$ and $j \in C_{1}$, and $a_{i j}$ for $i \in S_{j}$ and $j \in L_{2}$.

Example 9. (Atamtürk et al., 2001) p157. Consider the set $X^{G V U B}$

$$
\begin{array}{r}
x_{1}+x_{2}-x_{3}-x_{4} \leq 2 \\
x_{1} \leq 4+2 y_{1}+3 y_{2} \\
x_{2} \leq 3+y_{3}+2 y_{4} \\
x_{3} \leq 2+y_{5}+4 y_{6} \\
x_{4} \leq 4+2 y_{7}+2 y_{8} \\
x \in \mathbb{R}_{+}^{4}, y \in\{0,1\}^{8} .
\end{array}
$$

Taking $C_{1}=\{1,2\}, C_{2}=\emptyset, L_{2}=\{3,4\}, F=\{1,2,3\}$, we obtain $\lambda=a_{10}+$ $a_{20}+a_{4}-a_{30}-a_{40}-b=1$, and $\bar{a}=\max \{2,1,4,2,2\}=4$.

Taking as lifting function the MIR function $\phi(d)=\lambda F_{\alpha}\left(\frac{d}{\bar{a}}\right)$ with $\lambda=4$ and $\alpha=\frac{4-1}{4}$, we obtain $\phi(2)=0=\phi(3)=0$ and $\phi(4)=1$. The resulting inequality is

$\left(x_{1}-4-2 y_{1}-3 y_{2}\right)+\left(x_{2}-3-y_{3}\right)+(2-1)^{+}\left(1-y_{4}\right) \leq 8+y_{5}+y_{6}+y_{7}+y_{8}$. Using $G_{a, \lambda}$, as proposed in Proposition 15, leads to the same inequality.

In Atamtürk et al. (2001), a modified inequality is given for the case in which the different generalized VUBs have $0-1$ variables in common. This inequality can also be obtained directly by the lifted MIR procedure. 


\subsection{Generalized Upper Bounds}

Here we consider the set $X^{G U B}$

$$
\begin{aligned}
& \sum_{j \in N_{1}} x_{j}-\sum_{j \in N_{2}} x_{j} \leq b+s \\
& 0 \leq x_{j} \leq a_{j} y_{j} \text { for } j \in N_{1} \cup N_{2} \\
& \sum_{j \in S_{i}} y_{j} \leq 1 \text { for } i \in M_{1} \cup M_{2} .
\end{aligned}
$$

We assume for simplicity that the sets $S_{i}$ are disjoint and $S_{i} \subseteq N_{k}$ for $i \in M_{k}$ for $k=1,2$. This model has been examined by Wolsey (1990). In addition the knapsack set with GUBs has been studied in Gu et al. (1998) and lifted cover inequalities for such sets are used in Cplex and Xpress.

Definition 9. $C=\left(C_{1}, C_{2}\right)$ with $C_{k} \subseteq N_{k}$ for $k=1,2$ is a GUB-cover inequality if

i) $\left|C_{k} \cap S_{i}\right| \leq 1$ for $i \in M_{k}$ and $k=1,2$

ii) $\sum_{j \in C_{1}} a_{j}-\sum_{j \in C_{2}} a_{j}-b=\lambda>0$.

In addition we let $M_{k}^{+}=\left\{i \in M_{k}:\left|C_{k} \cap S_{i}\right|=1\right\}$, and $j(i) \in C_{k} \cap S_{i}$ be the unique element of $S_{i}$ in the cover, if any. For each $i$, select a subset $T_{i} \subseteq S_{i}$ such that $j(i) \in T_{i}$ for all $i \in M_{1}^{+} \cup M_{2}^{+}$.

Applying the MIR procedure, using 0-1 variables $z_{i}=\sum_{j \in T_{i}} y_{j}$ and their complements $\bar{z}_{j}=1-z_{j}$ for GUB sets in the cover, and taking the divisor $\bar{a}$ very large, we obtain a simple generalization of the GFC1 inequality (4).

Proposition 16. The inequality

$$
\begin{gathered}
\sum_{i \in M_{1}^{+}}\left[x_{j(i)}+\left(a_{j(i)}-\lambda\right)^{+}\left(1-y_{j(i)}\right)\right] \\
\left.\sum_{i \in M_{1}^{+}} \sum_{j \in T_{i} \backslash j(i)}\left[x_{j}-\left(\max \left(a_{j}, a_{j(i)}\right)-\lambda\right)^{+}\right) y_{j}\right] \\
\leq b+\sum_{j \in C_{2}} a_{j}+\sum_{i \in M_{2}^{+}} \sum_{j \in T_{i}} \min \left[\lambda, a_{j}-a_{j(i)}\right]^{+} y_{j} \\
+\sum_{i \in M_{2}^{+}} \sum_{j \in S_{i} \backslash T_{i}} x_{j}+\sum_{i \in M_{2} \backslash M_{2}^{+}} \sum_{j \in T_{i}} \lambda y_{j} \\
\sum_{i \in M_{2} \backslash M_{2}^{+}} \sum_{j \in S_{i} \backslash T_{i}} x_{j}
\end{gathered}
$$

is valid for $X^{G U B}$.

Other inequalities can be obtained using smaller values of $\bar{a}$.

Example 10. Consider the set $X^{G U B}$

$$
\begin{aligned}
& \begin{array}{llllll}
x_{1} & +x_{2} & +x_{3} & +x_{4} & -x_{5} & \leq 12
\end{array} \\
& \begin{array}{lllll}
y_{1} & +y_{2} & +y_{3} & & \leq 1
\end{array} \\
& x_{1} \leq 6 y_{1}, x_{2} \leq 7 y_{2}, x_{3} \leq 8 y_{3}, \quad x_{4} \leq 9 y_{4}, x_{5} \leq 6 y_{5} \\
& x \in \mathbb{R}_{+}^{5}, \quad y \in \quad\{0,1\}^{5} .
\end{aligned}
$$


¿From Proposition 16 with cover $C_{1}=\{2,4\}, C_{2}=\emptyset, \lambda=4$, and sets $T_{1}=$ $\{1,2,3\}$ and $T_{2}=\{4\}$, we obtain the valid inequality

$$
x_{2}+3\left(1-y_{2}\right)+x_{4}+5\left(1-y_{4}\right)+x_{1}-3 y_{1}+x_{3}-4 y_{3} \leq 12+4 y_{5} \text {. }
$$

Note that to carry out exact lifting for this model using the approach of Section 3, we need to use multi-dimensional lifting functions, unless each set of variables $S_{i}$ lies within just one of the vectors of variables $x^{k} \in X^{k}$ for some $k$.

\section{Conclusions}

In spite of the fact that we have concentrated in the last three sections on using MIR combined with simple superadditive lifting on a knapsack set with continuous variables to obtain strong valid inequalities for the various single node flow models, we believe that a more profound polyhedral study of the single node flow model $X^{N}$ is warranted, especially as most of the variants that have been studied can be viewed as faces and or projections of it.

The importance of superadditive lifting functions in permitting simultaneous lifting of sets of variables cannot be overestimated. Thus it seems crucial to improve our understanding of superadditive functions, as well as ways to calculate valid superadditive lifting functions. The fact that the submodularity of certain set functions arising from flows leads to valid inequalities with the simultaneous lifting property Wolsey (1989), and can be used to explain certain flow cover inequalities also appears to merit further investigation. As an example new inequalities for capacitated lot-sizing have been proposed recently Atamtürk and Munoz (2002) where certain coefficients can be derived using such submodular lifting.

Though we have not directly discussed computation with the inequalities presented here, various researchers have devised heuristic algorithms to choose the covers $C_{1}, C_{2}$ by solving some version of the knapsack problem

$$
\begin{gathered}
\min \sum_{j \in N_{1}^{\prime}}\left(1-x_{j}^{*}\right) z_{j}-\sum_{j \in N_{2}^{\prime}} x_{j}^{*} z_{j} \\
\sum_{j \in N_{1}^{\prime}} a_{j} z_{j}-\sum_{j \in N_{2}^{\prime}} a_{j} z_{j}>b \\
z \in\{0,1\}^{n_{1}^{\prime}+n_{2}^{\prime}}
\end{gathered}
$$

where $N_{i}^{\prime} \subseteq N_{i}$ for $i=1,2$ are suitably chosen subsets with $x_{j}^{*}$ not too far from its upper bound $a_{j} y_{j}^{*}$, see Gu et al. (1998); Van Roy and Wolsey (1987) and the discussion in Nemhauser and Wolsey (1988). Here it would undoubtedly be interesting to devise improved heuristics that take into account a priori part of the effects of lifting. Another possibility would be to test existing separation heuristics against an exact separation algorithm for $\operatorname{conv}\left(X^{N}\right)$.

Acknowledgement We are grateful to A. Atamturk and Y. Pochet for their helpful comments on an earlier version of this paper. 


\section{References}

A. Atamtürk. On the facets of single-constraint mixed-integer sets. Research Report BCOL.01.01, Department of Industrial and Operations Research, University of California at Berkeley, 2001.

A. Atamtürk. On capacitated network design cut-set polyhedra. Mathematical Programming, 92: $425-437,2002$.

A. Atamtürk and Munoz. A study of the lot-sizing polytope. Research Report BCOL.02.01, Department of Industrial and Operations Research, University of California at Berkeley, 2002.

A. Atamtürk, G.L. Nemhauser, and M.W.P. Savelsbergh. Valid inequalities for problems with additive variable upper bounds. Mathematical Programming, 91:145-162, 2001.

E. Balas. A time indexed formulation of non-preemptive single machine scheduling problems. Mathematical Programming, 8:146-164, 1975.

S. Ceria, C. Cordier, H. Marchand, and L.A. Wolsey. Cutting planes for integer programs with general integer variables. Mathematical Programming, 81:201-214, 1998.

H. Crowder, E.L. Johnson, , and M.W. Padberg. Solving large scale zero-one linear programming problems. Operations Research, 31:803-834, 1963.

M.X. Goemans. Valid inequalities and separation for mixed 0-1 constraints with variable upper bounds. Operations Research Letters, 8:315-322, 1989.

R.E. Gomory. An algorithm for the mixed integer problem. Research Report RM-2597, The Rand Corporation, 1960.

Z. Gu, G.L. Nemhauser, and M.W.P. Savelsbergh. Lifted flow cover inequalities for 0-1 integer programs: Computation. INFORMS J. of Computing, 10:427-437, 1998.

Z. Gu, G.L. Nemhauser, and M.W.P. Savelsbergh. Lifted flow cover inequalities for mixed 0-1 integer programs. Mathematical Programming, 85:439-468, 1999.

Z. Gu, G.L. Nemhauser, and M.W.P. Savelsbergh. Sequence independent lifting in mixed integer programing. J. of Combinatorial Optimization, 4:109-129, 2000.

P.L. Hammer, E.L. Johnson, and U.N. Peled. Facets of regular 0-1 polytopes. Mathematical Programming, 8:179-206, 1975.

R.G. Jeroslow. An introduction to the theory of cutting planes. Annals of Discrete Mathematics, 5: 71-95, 1979.

E.L. Johnson. Cyclic groups, cutting planes and shortest paths. In T.C. Hu and S.Robinson, editors, Mathematical Programming, pages 185-211. Academic Press, 1973.

H. Marchand and L.A. Wolsey. The 0-1 knapsack problem with a single continous variable. Mathematical Programming, 85:15-33, 1999.

H. Marchand and L.A. Wolsey. Aggregation and mixed integer rounding to solve mips. Operations Research, 49:363-371, 2001.

A. Miller, G.L. Nemhauser, and M.W.P. Savelsbergh. A multi-item production planning model with setup times: algorithms, reformulations, and polyhedral characterizations for a special case. Mathematical Programming B, 95:71-90, 2003a.

A. Miller, G.L. Nemhauser, and M.W.P. Savelsbergh. On the polyhedral structure of a multi-item production planning model with setup times. Mathematical Programming B, 94:375-406, $2003 \mathrm{~b}$.

G.L. Nemhauser and L.A. Wolsey. Integer and Combinatorial Optimization. John Wiley and Sons, 1988.

G.L. Nemhauser and L.A. Wolsey. A recursive procedure for generating all cuts for 0-1 mixed integer programs. Mathematical Programming, 46:379-390, 1990.

M. Oosten. A polyhedral approach to grouping problems. PhD thesis, University of Maastricht, 1996.

M. Padberg. On the facial structure of set packing polyhedra. Mathematical Programming, 5:199-215, 1973.

M.W. Padberg, T.J. Van Roy, and L.A. Wolsey. Valid inequalities for fixed charge problems. Mathematical Programming, 33:842-861, 1985.

J.-P.P. Richard, I.R. de Farias, and G.L. Nemhauser. Lifted inequalities for 0-1 mixed integer programming: Basic theory and algorithms. Research Report 2002, School of Industrial and Systems Engineering, Georgia Institute of Technology, 2002a.

J.-P.P. Richard, I.R. de Farias, and G.L. Nemhauser. Lifted inequalities for 0-1 mixed integer programming: Superlinear lifting. Research Report 2002, School of Industrial and Systems Engineering, Georgia Institute of Technology, 2002b.

T.J. Van Roy and L.A. Wolsey. Valid inequalities for mixed $0-1$ programs. Discrete Applied Mathematics, 14:199-213, 1986. 
T.J. Van Roy and L.A. Wolsey. Solving mixed 0-1 programs by automatic reformulation. Operations Research, 35:45-57, 1987.

J.I.A. Stallaert. The complementary class of generalized flow cover inequalities. Discrete Applied Mathematics, 77:73-80, 1997.

L.A. Wolsey. Faces for linear inequalities in 0-1 variables. Mathematical Programming, 8:165-178, 1975.

L.A. Wolsey. Facets and strong valid inequalities for integer programs. Operations Research, 24: $367-372,1976$

L.A. Wolsey. Valid inequalities and superadditivity for 0-1 integer programs. Mathematics of Operations Research, 2:66-77, 1977.

L.A. Wolsey. Submodularity and valid inequalities in capacitated fixed charge networks. Operations Research Letters, 8:119-124, 1989.

L.A. Wolsey. Valid inequalities for mixed integer programs with generalised upper bound constraints. Discrete Applied Mathematics, 25:251-261, 1990. 